\title{
A Unified Mechanism for Unconfined Deflagration-to-Detonation Transition in Terrestrial Chemical Systems and Type Ia Supernovae
}

\author{
Alexei Y. Poludnenko, ${ }^{1,2 *}$ Jessica Chambers,${ }^{3}$ Kareem Ahmed, ${ }^{3}$ \\ Vadim N. Gamezo, ${ }^{4}$ Brian D. Taylor ${ }^{5}$ \\ ${ }^{1}$ Department of Aerospace Engineering \\ Texas A\&M University, College Station, TX 77843, USA \\ ${ }^{2}$ Department of Mechanical Engineering \\ University of Connecticut, Storrs, CT 06269, USA \\ ${ }^{3}$ Department of Mechanical and Aerospace Engineering \\ University of Central Florida, Orlando, FL 32816, USA \\ ${ }^{4}$ Laboratories for Computational Physics and Fluid Dynamics \\ Naval Research Laboratory, Washington, DC 20745, USA \\ ${ }^{5}$ Munitions Directorate \\ Air Force Research Laboratory, Eglin, FL 32542, USA
}

*To whom correspondence should be addressed; E-mail: alexei.poludnenko@uconn.edu

A general theory of the deflagration-to-detonation transition is presented along with its experimental and numerical confirmation, as well as its application to Type Ia supernovae.

The nature of type Ia supernovae (SNIa) - thermonuclear explosions of white dwarf stars - is an open question in astrophysics. Virtually all existing theoretical models of normal, bright SNIa require the explosion to produce a detonation in order to consume all of stellar material, but the mechanism for the deflagration-to-detonation transition (DDT) remains unclear. We present a unified theory of turbulence-induced DDT that describes the mechanism and conditions for initiating detonation both in unconfined chemical and thermonuclear explosions. The model is validated using experiments with chemical flames and numerical simulations of thermonuclear flames. We use the 


\section{developed theory to determine criteria for detonation initiation in the single- degenerate Chandrasekhar-mass SNIa model, and show that DDT is almost inevitable at densities $10^{7}-10^{8} \mathbf{g ~ c m}^{-3}$.}

Type Ia supernovae (SNIa) are an important tool for measuring cosmological distances. Techniques used to calibrate SNIa for this purpose rely on empirical correlations (1-3) due to incomplete theoretical understanding of the mechanisms responsible for SNIa. Theoretical models of SNIa have remained limited due to uncertainties in the explosion mechanisms.

Explosions can involve two distinct types of combustion waves that differ by the propagation mechanism: deflagrations and detonations. Deflagrations, or flames, are relatively slow and propagate subsonically through heat conduction, diffusion, and advection. Detonations move supersonically due to ignition by shock compression, and therefore always involve strong shocks and propagate with the shock speed.

SNIa explosions are driven by fast thermonuclear burning in ${ }^{12} \mathrm{C} /{ }^{16} \mathrm{O}$ white dwarf (WD) stars with a mass close to, or below, the Chandrasekhar-mass limit of $\approx 1.4$ solar masses (4) - the maximum mass of a WD supported against the gravitational collapse by the electron degeneracy pressure. Beyond this general statement, however, the exact mechanisms of SNIa remain unclear (5-8), with a number of possible scenarios. These include the classical Chandrasekhar-mass $\left(\mathbf{M}_{c h}\right)$ model (9-12), sub-Chandrasekhar-mass (sub- $\mathbf{M}_{c h}$ ) models (13-16), double-degenerate (DD) models that include mergers or collisions of two WDs (17-20), and gravitationally confined detonations $(21,22)$.

All SNIa explosion scenarios share a common characteristic: to produce a normal, bright SNIa, detonation must be triggered in the stellar interior at some stage of the explosion. Such supersonic combustion is required to consume the entire WD, including its outer layers, which expand supersonically as the material becomes gravitationally unbound. Observations place tight upper limits on the amount of unburned ${ }^{12} \mathrm{C}$ in the resulting SNIa ejecta indicating nearly complete combustion of the stellar material in the explosion $(23,24)$. Pure deflagration $\mathbf{M}_{c h}$ explosion scenarios have been previously suggested as the mechanism forming subluminous classes of supernovae, e.g., Type Iax (25).

Generally, it is more difficult to ignite a detonation than a flame in a chemically or thermonuclearly explosive mixture. Detonation can typically form in one of three ways: i) strong ignition, in which a detonation is directly initiated by a pre-existing, or externally introduced, strong shock (26); ii) weak ignition, in which a detonation develops via a spontaneous reaction wave propagating through a gradient of temperature or composition $(27,28)$ created by weaker shocks or other processes (gradient mechanism); and 3) deflagration-to-detonation transition (DDT), during which shocks are produced by fast flames and the detonation may sometimes form at the final stages via the gradient mechanism (29).

While strong ignition may be realized in the gravitationally confined model $(21,22)$ or in the case of WD collisions $(20,30)$, more widely accepted models, such as the $\mathbf{M}_{c h}$ and sub- $\mathbf{M}_{c h}$ scenarios, as well as the DD mergers, generally do not have any natural mechanism to form preexisting shocks of sufficient strength to directly ignite a detonation. For example, in the classical 
$\mathrm{M}_{c h}$ scenario, detonation formation requires a DDT because burning must initially propagate via subsonic flames to pre-expand a star and produce intermediate-mass elements $(9,31,32)$. In the sub- $\mathrm{M}_{c h}$, both the gradient and the DDT mechanisms of detonation formation cannot be ruled out. Material compression produced by accretion in the surface layers leads to ignition, which could produce either: localized hotspots directly triggering a detonation through the gradient mechanism; or a flame, which could subsequently trigger a DDT. We focus on the DDT in degenerate stellar matter as the potential mechanism of detonation ignition in the two leading SNIa scenarios, namely $\mathbf{M}_{c h}$ and sub-M $\mathbf{M}_{c h}$. Although, DDT is generally not considered in the context of the DD models, the detailed ignition process in that scenario remains unclear.

Thermonuclear combustion waves are qualitatively similar to chemical combustion waves on Earth, as they are controlled by the same physical mechanisms. This similarity allows us to seek insights into the fundamental aspects of the physical processes controlling SNIa explosions using theoretical, numerical, and experimental results obtained for terrestrial chemical systems. DDT is also relevant to terrestrial applications ranging from detonation-based propulsion and power-generation systems, e.g., detonation engines (33-37), to the industrial safety of mining operations (38), fuel-storage, chemical processing $(39,40)$, and nuclear power-generation facilities (41). Large-scale industrial accidents in Buncefield, UK $(42,43)$ (fuel-storage facility, 2005), Sago Mine, US (44) (coal mine, 2006), Jaipur, India (45) (chemical processing plant, 2009), and Fukushima, Japan (46) (nuclear power plant, 2011) may all have involved a DDT.

The DDT process in SNIa occurs in essentially unconfined conditions, in the sense that there are no walls or obstructions that are usually present in terrestrial settings. The mechanism of such unconfined DDT is not understood in chemical systems on Earth. Direct numerical simulations (DNS) have shown that chemical flames interacting with high-intensity turbulence can spontaneously accelerate and produce strong shocks or detonations in a completely unconfined setting (47). We present an experimental confirmation of this shock-generation and DDT mechanism in terrestrial systems, and apply it to SNIa.

\section{Theory of turbulence-induced DDT}

DNS of chemical flames in high-intensity turbulent flows have shown that turbulent flames with burning speeds $S_{T}$ above the speed of a Chapman-Jouguet (CJ) deflagration, $S_{C J}$, are intrinsically unstable and can transition to detonations (47). The $S_{C J}$ is defined as the flame speed, at which the flow on the product side of the flame becomes sonic in the reference frame co-moving with the flame. Consequently, the condition for the turbulence-driven spontaneous DDT (tDDT) can be written as (47)

$$
S_{T}>S_{C J}=c_{s} / \alpha
$$

where $c_{s}$ is the sound speed in hot products, and $\alpha=\rho_{f} / \rho_{p}$ is the ratio of the densities of fuel $\rho_{f}$ and combustion products $\rho_{p}$.

Once the flame speed exceeds $S_{C J}$, transition to a detonation occurs as a catastrophic runaway process, which results in a rapid pressure build-up and creates strong shocks inside the 
turbulent flame. The evolution of a chemical turbulent flame in this process and its ultimate transition to a detonation are shown in Fig. 11 \& Movie S1 (this simulation was previously reported elsewhere (47)). The corresponding histories of the turbulent flame speed and maximum pressures in the domain are shown in Fig. $2 \mathrm{C}$.

This condition stems from the fact that $S_{C J}$ is the maximum possible speed of a steady-state deflagration, which satisfies conservation laws (48). The actual flame speed $S_{T}$ depends on the turbulent intensity and the turbulent-flame structure, and can exceed $S_{C J}$. Because conservation laws prohibit a steady-state deflagration wave for $S_{T}>S_{C J}$, the flow would evolve producing shocks and accelerating, potentially reaching a new steady state permitted by the conservation laws. As a result, the flame could either become pulsatingly unstable (49) periodically oscillating between the speed above and below $S_{C J}$ and producing shocks, or it could transition to a CJ detonation, which can also be viewed as a CJ deflagration coupled to a shock.

Equation 1 is equivalent to (47)

$$
\dot{e} \gtrsim e / t_{s}
$$

where $e$ is the internal (thermal) energy, $\dot{e}$ is the energy release rate in a flame, and $t_{s}=\delta_{T} / c_{s}$ is the sound crossing time of a turbulent flame with width $\delta_{T}$. Equation 2 essentially provides the physical meaning for Equation 1 ; when the flame speed exceeds the CJ threshold, burning releases within a sound-crossing time the amount of energy close to, or greater than, the internal energy stored in the flame volume. This results in a build-up of pressure, and ultimately causes the formation of strong shocks.

The criterion defined by Equation 1 does not predict the onset of tDDT in a given turbulent reactive flow. Instead, we need to determine the corresponding critical turbulent conditions, at which the turbulent flame speed can reach $S_{C J}$ and thus allow the turbulence-induced pressure runaway to occur.

The tDDT process can occur while the flame remains in the flamelet regime, defined as the combustion regime in which the turbulent flame can be viewed as a folded laminar flame sheet with the internal structure minimally affected by turbulence (47). Other theoretical models (5053) have suggested that turbulence-driven DDT requires formation of distributed flames, and thus necessitates higher turbulent intensities capable of disrupting the internal flame structure. However, the tDDT mechanism, which triggers the pressure runaway and ultimately DDT, is not dependent on the structure of a turbulent flame or the particular combustion regime as long as Equation 1 is satisfied. This process can be observed even in idealized one-dimensional flames by artificially increasing the flame speed (54). Therefore, a similar set of critical turbulent conditions can be obtained also for distributed flames. We focus on the derivation of the critical turbulent conditions in the flamelet regime.

In the flamelet regime, the turbulent flame speed $S_{T}$ is (55)

$$
S_{T}=I_{M} S_{L} \frac{A_{T}}{L^{2}}
$$

where $A_{T}$ is the surface area of the flame sheet folded in a volume of size $L, S_{L}$ is the laminar 
flame speed, and the coefficient $I_{M}$ accounts for the effects of turbulent stretch. For thermonuclear flames, $I_{M}$ is of order unity (56).

In the volume $L^{3}$, turbulence would fold the flame on all scales greater than some small scale $\lambda_{f}$, on which the turbulent intensity $U_{\lambda} \equiv U(\lambda)$ can be estimated as

$$
U_{\lambda}=\alpha I_{M} S_{L}
$$

As a result of such packing, the flame surface density in a unit volume is equal to the inverse of the average flame-sheet separation, which is effectively the scale of the smallest flame folds $\lambda_{f}$

$$
\frac{A_{T}}{L^{3}}=\frac{1}{\lambda_{f}}
$$

Based on the criterion in Equation 1, at the onset of the runaway, $S_{T}=c_{s} / \alpha$. Substituting this along with Equation 5 into Equation 3 , we can find the characteristic flame volume $L_{C J}$, in which the flame can achieve the CJ deflagration conditions

$$
L_{C J}=\lambda_{f} \frac{c_{s}}{\alpha I_{M} S_{L}} .
$$

In particular, the smallest possible size of the flame region, in which tDDT can occur, $L_{C J}^{\min }$, corresponds to the maximally tight flame packing, in which the average separation of flamesheets, $\lambda_{f}$, is close to the laminar flame thickness, $\delta_{L}$,

$$
L_{C J}^{\min }=\delta_{L} \frac{c_{s}}{\alpha I_{M} S_{L}} .
$$

We assume that turbulent properties of the reacting flow field can be effectively represented by homogeneous isotropic Kolmogorov-type turbulence. In reality, the presence of exothermic reactions can change the turbulence structure inside the flame (57-59). These changes are primarily driven by the fluid expansion as well as the increase of the temperature-dependent viscosity, and such effects can be pronounced in chemical flames. In contrast, in thermonuclear flames, their impact is minimal due to very low density ratios across the flame $\alpha \lesssim 2$ and extremely small ratios of viscosity to thermal conduction in degenerate plasmas defined via the Prandtl number $\operatorname{Pr} \sim 10^{-5}(60)$.

Using Kolmogorov scaling, we can find turbulent intensity $U_{l}^{\max }$ at a characteristic integral scale, $l$, which would produce such maximally tight flame packing corresponding to Equation 7

$$
U_{l}^{\max }=\alpha I_{M} S_{L}\left(\frac{l}{\delta_{L}}\right)^{1 / 3}
$$

Here we used Equation 4 as the turbulent intensity at the scale $\delta_{L}$. This integral turbulent velocity would provide the maximum burning rate per unit volume as it would create the most tightly packed flame configuration. Therefore, it would create conditions for the onset of tDDT in the smallest possible volume. 
At the same time, CJ conditions can also be realized at lower turbulent intensities $U_{l}<$ $U_{l}^{\max }$. In this case, the smallest flame folds will occur on scales $\lambda_{f}>\delta_{L}$, and the resulting critical flame volume $L_{C J}$ required to achieve the CJ burning speed (Equation 6) would be larger than $L_{C J}^{\min }$. In particular, turbulent intensity $U_{C J}$ at the scale $L_{C J}$, which would provide sufficient flame packing on that scale to achieve $S_{T}=S_{C J}$, can be found as

$$
U_{C J}=U_{\lambda}\left(\frac{L_{C J}}{\lambda_{f}}\right)^{1 / 3} .
$$

Using Equation 4 and 6 , this gives

$$
U_{C J}=\left(\alpha I_{M} S_{L}\right)^{2 / 3} c_{s}^{1 / 3} .
$$

This turbulent intensity is independent of scale and only depends on the properties of the reactive mixture. Therefore, we can use Equation 10 and the Kolmogorov scaling to express $L_{C J}$ in terms of the characteristic turbulent integral scale, $l$, and velocity, $U_{l}$, in a given system

$$
L_{C J}=\left(\alpha I_{M} S_{L}\right)^{2} c_{s} \frac{l}{U_{l}^{3}} .
$$

The critical flame speed $S_{T}=S_{C J}$ at the scale $L_{C J}$ can be, and typically is, higher than the turbulent velocity $U_{l}=U_{C J}$ at that scale (see Fig. 2). Thus, such $S_{T}$ does not represent the burning speed of a flame in equilibrium with the ambient turbulent flow field. For $S_{T}>U_{l}$, the upstream turbulence cannot supply fresh fuel to the turbulent flame sufficiently fast to sustain such high burning rates, and faster burning can be supported only by the fuel already contained within the flame. Such unsteady configurations can form either when a flame enters the region of sufficiently fast turbulence, or when turbulent intensity gradually increases above the critical values given by $U_{C J}$ or $U_{l}^{\max }$. Ultimately, this unsteady burning continues as a runaway process either until a detonation is formed, or the fuel in the flame burns out, which can lead to the pulsating instability of turbulent flames associated with the periodic formation of shocks (49).

Equations 7, 8, 10, and 11 provide the criteria for the onset of the pressure runaway, at which $S_{T}=S_{C J}$. In this sense, they represent only the necessary conditions for the detonation initiation. The sufficiency condition, which defines whether a shock or a detonation form as a result of the pressure runaway, depends on the duration of this process. If the fuel in the turbulent flame burns out within a sound crossing time of the flame, the result is equivalent to a constant-volume explosion, which generates pressures insufficient for a detonation initiation (both in chemical and thermonuclear mixtures). On the other hand, if the burn-out lasts for several sound crossing times, pressure will build up on the upstream side of the turbulent flame due to the gradient of the sound speed in the flame. Such gradual pressure accumulation will ultimately produce shocks of sufficient strength to ignite a detonation.

For packed flame configurations, the ratio of the burn-out time $t_{B}=\lambda_{f} /\left(I_{M} S_{L}\right)$ of the flame to the sound-crossing time $t_{s}=L_{C J} / c_{s}$ is equal only to the flame density ratio

$$
\frac{t_{B}}{t_{s}}=\alpha,
$$


which follows from Equation 6. Packed configurations formed by terrestrial chemical flames with $\alpha \sim 3-10$ (48) burn out during several sound-crossing times. This allows for the pressure accumulation on the upstream side of the flame and creates conditions for shock amplification, capable of producing DDT (cf. Fig. 1F, G, H). For thermonuclear flames in degenerate plasmas, however, $\alpha=1.2-2.0$ (60), and the burn-out occurs during only one or two sound-crossing times. The resulting shocks are similar to those produced in a constant-volume explosion and are of insufficient strength to ignite detonations directly. These shocks, however, can further amplify by propagating in the density gradient of a star (61), or by interacting with surrounding turbulent flames on larger scales. We thus consider Equation 1 as the criterion for DDT in SNIa, although the DDT mechanism in SNIa requires an additional step for shock amplification to DDT strengths.

These analytical results are consistent with the DNS of chemical flames (47). $L_{C J}^{\mathrm{min}}$ is very close to the domain size in calculations where pressure runaway was observed (47). Those DNS relied on a simplified physical model involving an ideal-gas equation of state and a single-step, first-order Arrhenius chemical kinetics representing $\mathrm{H}_{2}$-air- and $\mathrm{CH}_{4}$-air-like mixtures. Thus comparison with those DNS does not determine whether this mechanism of tDDT would also be present in realistic chemical mixtures. In order to address this issue, next we present results of an experimental study demonstrating the tDDT process in turbulent $\mathrm{H}_{2}$-air flames propagating with super-CJ speeds.

\section{Experimental study}

During a SNIa explosion, DDT would occur in unconfined conditions, i.e., in the absence of walls or obstructions that could confine pressure thus creating conditions for its build-up. It is difficult to create a similar experimental setting, in which the evolution of a perfectly unconfined flame can be observed from ignition to the onset of a detonation. However, even in a confined experimental configuration, it is possible to isolate the turbulence-flame coupling, which could lead to a pressure runaway and a DDT, from other phenomena related to the interactions of high-speed flows with obstructions or turbulent boundary layers. We designed an experiment to test this.

A schematic diagram of the Turbulent Shock Tube (TST) facility and the resulting flow structure are shown in Figs. 3 and 4. This facility is intended to create high-speed turbulent conditions, which can lead to the spontaneous flame acceleration and tDDT that were previously modeled in DNS $(47,49)$. The TST consists of a $1.5 \mathrm{~m}$ long channel with one open and one closed end, with a square cross section of $45 \times 45 \mathrm{~mm}^{2}$. A spark plug is mounted at the center axis of the channel at the closed end and used to ignite the flame. A test section is $152 \mathrm{~mm}$ long with optical access on three sides, which creates a visibility domain of approximately $145 \times$ $45 \mathrm{~mm}$ for advanced flame and flow-field diagnostics (62). The diagnostic section windows are composed of $25 \mathrm{~mm}$ thick fused silica designed to sustain high pressures associated with detonation waves. Premixed hydrogen-air mixtures with varying composition are used. 
Upon ignition, the flame kernel initially expands to fill the entire cross-sectional area of the channel. Once the flame front has developed, it begins to propagate toward the open end of the channel. A series of five perforated plates are positioned inside the facility close to the point of ignition to generate turbulence in the flow passing through them and thus to produce rapid flame acceleration. As a result, a leading shock wave with Mach $\sim 2-3$ is formed ahead of the flame. After this shock passes through the last perforated plate immediately before the diagnostic section, the post-shock flow creates multiple high-speed jets that produce high levels of turbulence within the reactants. The turbulent intensity is controlled by the equivalence ratio, $\phi$, of the initial mixture. Higher values of $\phi$ result in faster laminar flame speeds and more rapid initial flame acceleration, which translates into larger leading shock velocities and higher turbulence levels. The geometric configuration employed allows us to survey the flame regimes of interest and focus on the conditions for tDDT. Extensive experimental testing of the design and arrangement of perforated plates has been performed to ensure that desired turbulence conditions are achieved, specifically in terms of the amplitude of turbulent velocity fluctuations (63-65).

After the flame passes the last perforated plate and emerges in the diagnostic section, it starts to interact with the high-speed turbulence in this section. Subsequent flame evolution depends on the flame initial burning velocity, $S_{T}$, which is determined by the level of turbulent fluctuations. Depending on the value of $S_{T}$ relative to the CJ deflagration speed, the flame may or may not produce strong shocks and undergo the tDDT. In this setup, varying mixture composition over a very narrow range of $\phi$ allows us to probe the flame dynamics, as well as the details of the tDDT process, as $S_{T}$ is increased from below to above the CJ threshold.

The turbulent flame evolution in the experiments is shown in Fig. $4 \mathrm{~F}-\mathrm{J}$ in a sequence of schlieren images, representing flow density gradients manifested via the gradients of the refractive index. Two experiments are shown with mixture equivalence ratios of $\phi=0.888$ (panels F-H) and $\phi=0.905$ (panels I, J) to highlight different stages of tDDT. Figure 4A-E also shows synthetic schlieren images obtained from the DNS (see Fig. 1) (47) at the similar stages of the flame evolution. Synthetic schlieren images represent $\log (\nabla \rho)$ computed in a 3D volume and projected onto a 2D plane. Although an $\mathrm{H}_{2}$-air mixture was used in the experiments, the DNS shown in Figs. 1 and 4 used a $\mathrm{CH}_{4}$-air mixture, which demonstrates the independence of the results on the choice of a reactive mixture. The laminar flame speed in $\mathrm{CH}_{4}$-air is almost an order of magnitude lower than in $\mathrm{H}_{2}$-air $\left(38 \mathrm{~cm} \mathrm{~s}^{-1}\right.$ vs. $302 \mathrm{~cm} \mathrm{~s}^{-1}$, respectively). Calculations using $\mathrm{H}_{2}$-air mixture have been discussed elsewhere (47). At the time shown in Fig. $4 \mathrm{~F}-\mathrm{J}$, boundary layers have not developed and so their influence on the observed flame acceleration is negligible.

Figure 4 shows similar dynamics in the experiment and the DNS. Pressure waves generated within the turbulent flame propagate into the unburned material and form a compressed region ahead of the flame (Fig. 4B,G). As the runaway process develops, multiple pressure waves coalesce into a flame-generated shock, the strength of which grows with time (Fig. 4C,H). This shock forms between the flame and the leading shock that was transmitted through the last perforated plate. Eventually, the shock approaches the von Neumann (post-shock) pressure of a CJ detonation (also see Fig. 2A), at which point it triggers a DDT (Fig. 4D,I) and forms a 
detonation (Fig. 4E,J). The case with $\phi=0.905$ (Fig. 4I,J) results in a detonation, but the corresponding peak pressure shown in Fig. $2 \mathrm{~A}$ is lower than $P_{C J}$. This is because pressures reported in Fig. 2/A were measured in the first diagnostic window, while DDT occurred in the second window.

Figure 2 A shows peak pressures in the leading shock and those produced by the flame, along with the corresponding turbulent flame speeds, as a function of the equivalence ratio in multiple experiments. The evolution of the pressure build-up inside the turbulent flame and the emergence of a flame-generated shock for a specific experiment with $\phi=0.888$ are demonstrated in Fig. 2B. It provides pressure histories recorded by several pressure transducers shown in Fig. 3 .

Figure 2A shows that the flame starts to generate strong pressure waves when its burning velocity $S_{T}$ exceeds the $S_{C J}$ threshold, i.e., when Equation 1 is satisfied. This results in the formation of a compressed region of high pressure immediately ahead of the flame (Figs. $4 \mathrm{~F}-\mathrm{H}$ ). Figure $2 \mathrm{~B}$ shows that pressure growth is associated with the turbulent flame and not supported by the closed end of the channel. Pressure in the vicinity of the flame as recorded by transducers increases by $\approx 70 \%$. This occurs as the flame propagates down the length of the channel and passes transducers located progressively further downstream (Fig. 3). The pressure recorded by transducer $\# 1$ at later times drops and eventually plateaus at a level well below the peak pressures recorded in the flame region. The pressure recorded at later times by transducer \#4, which is located further ahead of transducer \#1 (cf. Fig. 2), exhibits the same decreasing trend and reaches values very close to those recorded by transducer \#1. This shows that pressure upstream of the flame front close to the last perforated plate does not change with time, which would be the case if the observed increase in pressure in the flame region was the result of pressurization of the entire flow from the flame to the closed end of the channel.

Figure 2A demonstrates that the turbulent flame speed in the experiments shown in Fig. 4 satisfies Equation 1. We now consider whether turbulent conditions in these experiments are in agreement with the theory presented above. In particular, at $\phi=0.888$, turbulent integral velocity, $U_{l}$, ahead of the flame at the beginning of the diagnostic section, i.e., at the start of the runaway process resulting in the pressure build-up, had an average value of $68.85 \mathrm{~m} \mathrm{~s}^{-1}$ with the maximum and minimum values of $238.56 \mathrm{~m} \mathrm{~s}^{-1}$ and $9.70 \mathrm{~m} \mathrm{~s}^{-1}$, respectively, and a standard deviation of $37.68 \mathrm{~m} \mathrm{~s}^{-1}$. Values of the turbulent integral scale, $l$, were $1.45 \mathrm{~cm}$ (average), 3.17 $\mathrm{cm}$ (maximum), $0.19 \mathrm{~cm}$ (minimum), and $0.45 \mathrm{~cm}$ (standard deviation). Procedures for determining these turbulent characteristics in the TST facility have been described elsewhere (65). The pressure and temperature of the reacting mixture in the compressed region ahead of the flame are $6.92 \mathrm{~atm}$ and $627 \mathrm{~K}$, respectively. These allow us to determine the corresponding laminar flame properties and $S_{C J}$. The critical turbulent integral velocity $U_{l}^{\max }$ defined in Equation 8 and corresponding to the average integral scale in the flow is $166.76 \mathrm{~m} \mathrm{~s}^{-1}$. This turbulent velocity would be required to provide maximally tight flame packing. It is, however, larger than the average $U_{l}=68.85 \mathrm{~m} \mathrm{~s}^{-1}$ in the flow, which implies that flame would be folded on scales larger than $\delta_{L}$. As a result, the flame volume required to reach the $\mathrm{CJ}$ conditions would be larger than $L_{C J}^{\min }$ (Equation 7) and instead would be defined by Equation 6, namely $L_{C J}=2.6 \mathrm{~cm}$, which is within the range of values of $l=0.19-3.17 \mathrm{~cm}$ observed in the experiment and $80 \%$ 
larger than the average $l=1.45 \mathrm{~cm}$. The corresponding $U_{C J}=83.64 \mathrm{~m} \mathrm{~s}^{-1}$ (Equation 10 is within $20 \%$, and less than $\sigma$, of the average $U_{l}=68.85 \mathrm{~m} \mathrm{~s}^{-1}$ in the experiment. Therefore, theoretically predicted turbulent conditions for the onset of the pressure runaway are within the range of values observed in the experiment.

Similar analysis can be carried out for the DNS shown in Fig. 4. Turbulent integral velocity and scale in the calculation are $U_{l}=22.37 \mathrm{~m} \mathrm{~s}^{-1}$ and $l=0.31 \mathrm{~cm}$, respectively. Despite the large turbulent intensities in this calculation, only the preheat zone of the flame, where the heat and products diffuse into the cold reactants, is broadened (57). Thus, overall, combustion proceeds in the flamelet regime, which is consistent with the theory developed above. Using the laminar-flame properties for the single-step $\mathrm{CH}_{4}$-air reaction model (66), the corresponding $U_{C J}=19.25 \mathrm{~m} \mathrm{~s}^{-1}$ and $L_{C J}=0.2 \mathrm{~cm}$, which are just below $U_{l}$ and $l$ in the calculation. Therefore, turbulent conditions in this calculation are also in agreement with the theoretical predictions for the onset of the pressure runaway.

\section{Numerical modeling in thermonuclear systems}

The equation of state, transport properties, and chemical kinetics that characterize degenerate thermonuclear plasma in a WD interior during a SNIa explosion differ in several aspects from those representative of chemical reactive systems. Thermonuclear deflagrations are characterized by very low density ratios, $\alpha \sim 1.2-2$ (60), in contrast with chemical flames, in which $\alpha \sim 3-10$ (48). Because the formation of a sonic point in a subsonic CJ deflagration is determined by the fluid expansion in a flame, lower density ratios in thermonuclear plasmas could affect the overall dynamics of turbulent super-CJ deflagrations. Therefore, we next test our theoretical model under appropriate conditions of burning thermonuclear plasmas.

Numerical simulations allow only a limited range of scales to be modeled in a typical calculation of turbulent flames. To capture the tDDT process in a simulation, $L_{C J}^{\min }$ for a given reactive mixture must be sufficiently small to be accommodated in a limited computational domain.

We considered a variety of mixture compositions, from pure ${ }^{4} \mathrm{He}$ and ${ }^{4} \mathrm{He} /{ }^{12} \mathrm{C}$ mixtures, which would represent sub- $\mathrm{M}_{c h}$ and DD models, to ${ }^{12} \mathrm{C} /{ }^{16} \mathrm{O}$ mixtures relevant to the $\mathrm{M}_{c h}$ scenario. We found that $L_{C J}^{\min }$ becomes sufficiently small to be simulated, namely $L_{C J}^{\min } / \delta_{L} \lesssim 100$, only for pure ${ }^{12} \mathrm{C}$ mixtures at high densities $\rho \gtrsim 2 \times 10^{8} \mathrm{~g} \mathrm{~cm}^{-3}$ (62). While DDT would not be expected to occur at such densities in a SNIa, this nevertheless allows us to simulate tDDT in a degenerate plasma undergoing thermonuclear burning.

We model thermonuclear flame dynamics using compressible reactive-flow equations solved with a finite-volume code ATHENA-RFX $(67,68)$. Further details of the modeling approach are given in (62).

For the thermonuclear-flame simulation, we use a flame-in-a-box computational setup similar to the previous chemical-flame DNS (47) shown in Fig. 1, which has also been used in prior simulations of thermonuclear flames (69-72). Such calculations based on first principles represent a small region of the flow inside a WD allowing us to fully resolve the flame and its 
evolution in the turbulent flow. The flame interacts with a homogeneous, isotropic upstream turbulence steadily driven at the scale of the domain width using a spectral method, which introduces divergence-free velocity fluctuations into the flow, with a prescribed energy injection spectrum and rate (68). This approach ensures that the turbulent integral velocity, $U_{l}$, and scale, $l$, in the upstream flow are nearly constant both in space and time with a standard deviation of $\lesssim 2 \%$ and $\lesssim 5 \%$, respectively. An analysis of the resulting turbulence, both reacting and nonreacting, including comparison with prior experimental and DNS results, has been presented elsewhere $(57,58)$.

The domain size and turbulent intensity were set based on the criteria given in Equations 7 - 10 (see Table S1). The calculation uses a rectangular domain with width equal to the minimal CJ system size $L_{C J}^{\min }=0.02 \mathrm{~cm}$ (Equation 7) for the fuel density $\rho=4 \times 10^{8} \mathrm{~g} \mathrm{~cm}^{-3}$. At this density, the laminar flame speed $S_{L}=1.35 \times 10^{7} \mathrm{~cm} \mathrm{~s}^{-1}$ and the CJ speed $S_{C J}=4.8 \times 10^{8}$ $\mathrm{cm} \mathrm{s}^{-1}$, or respectively $\approx 2 \%$ and $\approx 70 \%$ of the sound speed in fuel. The calculation is performed on a uniform Cartesian mesh with a cell size $d x=3.91 \times 10^{-5} \mathrm{~cm}$, which corresponds to $\delta_{L} / d x=4.68$. This resolution is sufficient to capture the laminar flame properties and is similar to that used in prior studies of fully-resolved thermonuclear deflagrations $(71,72)$. Initially, turbulence is allowed to evolve for $\approx 5.2 \tau_{e d}$ in order to reach an equilibrium steady state, where $\tau_{e d}=l / U_{l}$ is the integral-scale eddy turnover time. The resulting turbulence is characterized by the integral velocity $U_{l}=8 \times 10^{7} \mathrm{~cm} \mathrm{~s}^{-1} \approx 1.84 U_{C J}$ (Equation 10 ) or $\approx 12 \%$ of the sound speed in fuel, so $U_{l}<S_{C J}$. The corresponding turbulent integral scale $l \approx L_{C J}^{\min } / 4$. At the time $t=5.2 \tau_{e d}$, the thermodynamic state in the domain is re-initialized with the exact laminar flame solution corresponding to the initial fuel temperature $10^{8} \mathrm{~K}$. Boundary conditions are periodic in the spanwise direction (transverse to the direction of flame propagation) and zero-order extrapolation (outflow) along the flame-propagation direction, which allows products to flow out from the back of the domain and does not create a confining effect capable of promoting pressure build-up. Despite the substantial level of turbulent intensity, turbulent energy dissipation has a negligible effect on the system dynamics. By the end of the calculation, fuel temperature increases to $\approx 8.5 \times 10^{8} \mathrm{~K}$ from an initial $1.0 \times 10^{8} \mathrm{~K}$. As a result, the laminar flame speed increases by $\lesssim 8 \%$.

Figure $2 \mathrm{D}$ shows the evolution of $S_{T}$ and maximum pressure in the domain in this calculation. The burning speed increases, rapidly exceeding $S_{C J}$ and approaching the detonation speed $D_{C J}$. Once $S_{T}$ exceeds $U_{l}$, however, the flame decouples from the upstream turbulence. It rapidly consumes the fuel ingested into the flame during the early stages of the evolution, completing the burn-out within approximately one sound crossing time, in agreement with Equation 12. Pressure growth accelerates once $S_{T}$ crosses the $S_{C J}$ threshold, similar to the behavior observed in chemical mixtures (Fig. 2A-C). Eventually, a strong shock forms, exits the flame, and propagates into the fuel upstream. Figure 5 shows the corresponding flame structure at the time of maximum $S_{T}$ (panel A) and after the completion of the flame burn-out (panel B), as well as the structure of the resulting shock wave (panel B) (also see movie S2). The resulting shock has local peak pressures $\approx 2 \times 10^{26} \mathrm{erg} \mathrm{cm}^{-3}$ (Fig. 5B), and the spanwise-averaged peak pressures $\approx 1.85 \times 10^{26} \mathrm{erg} \mathrm{cm}^{-3}$ (cf. Fig. 2D). The corresponding shock Mach number 
$M_{s} \approx 1.15$. This shock is stronger than the one produced in a constant-volume explosion at these plasma conditions, for which the peak pressure $P_{C V}=1.73 \times 10^{26} \mathrm{erg} \mathrm{cm}^{-3}$ and Mach number $M_{C V}=1.09$ (62).

Thus similar to chemical mixtures, the interaction of a highly subsonic thermonuclear flame with a highly subsonic turbulence can produce strong shocks in unconfined degenerate plasmas. A rapid runaway process resulting in pressure build-up occurs once the flame speed exceeds the critical CJ deflagration threshold in agreement with Equation 1. The turbulent conditions derived above, namely, $L_{C J}^{\min }$ (Equation 7 ) and $U_{C J}$ (Equation 10, also match the onset of the runaway in degenerate plasmas. This suggests that the overall mechanism is applicable to both chemical and thermonuclear mixtures.

We next investigate whether shocks, which are produced in the process described above, can ultimately trigger a detonation. Such shocks can further amplify by propagating in the density gradient of a star (61), or by interacting with surrounding turbulent flames; we focus on the latter mechanism.

While the strength of the shock observed in the DNS was larger than in a constant-volume explosion, we assume conservatively that shocks produced by super-CJ flames in thermonuclear plasmas are close to those in constant-volume explosions. Propagating such a shock through a 3D turbulent flame until detonation ignites is computationally expensive. Therefore, we demonstrate this process in a $2 \mathrm{D}$ calculation, in which a turbulent flame is represented by a series of spherical flames. An additional benefit of considering this problem in $2 \mathrm{D}$ is that we can model this process at a density $\rho=3 \times 10^{7} \mathrm{~g} \mathrm{~cm}^{-3}$ and in a realistic $50 / 50{ }^{12} \mathrm{C} /{ }^{16} \mathrm{O}$ composition, which are closer to the DDT conditions expected in SNIa. We consider the propagation of a constantvolume explosion shock with Mach number $M_{s}=1.28$ and $P_{C V}=7.1 \times 10^{24} \mathrm{erg} \mathrm{cm}^{-3}$ through a series of spherical flames with diameter $10 \delta_{L}$ and separation between sphere centers $30 \delta_{L}$. Such a configuration represents a loosely packed flame. The calculation is performed on a uniform grid with resolution $\Delta x=0.0257 \mathrm{~cm}$ or 4.4 cells per half- ${ }^{12} \mathrm{C}$-reaction zone length of a CJ detonation, defined as the distance from the shock to the point at which the mass fraction of ${ }^{12} \mathrm{C}$ reaches half its maximum value. Similar resolution was used in prior detonation studies, where it was shown to reproduce the detonation velocity and resolve the characteristic multidimensional cellular structure of unstable thermonuclear detonations (73). The size of such detonation cells is $\approx 3 \mathrm{~cm}$ (73) resulting in $\approx 2.4$ detonation cells in the $7.2 \mathrm{~cm}$ wide channel. The corresponding resolution of a laminar flame is 9.3 computational cells per $\delta_{L}$. The initial flame configuration is shown in the upper panel of Fig. 6 .

As the shock begins to propagate through the turbulent flame, it compresses the flame and generates more flame surface. Both processes accelerate burning, which ultimately results in a pressure increase that couples to the shock and amplifies it. This process is illustrated by the time sequence of frames in Fig. 6. Figure 7A shows the pressure distribution through the domain at several times. As the shock accelerates, the pressure rapidly approaches the von Neumann value, at which point the detonation is ignited. The resulting detonation speed is $1.16 \times 10^{9}$ $\mathrm{cm} \mathrm{s}^{-1}$, which is equal to the ideal speed of a freely propagating CJ detonation.

Figure $7 \mathrm{~B}$ summarizes the results of several $2 \mathrm{D}$ simulations performed for the same flame 
configuration but different initial shock Mach numbers $M_{s}$. These show the dependence of the distance and time to DDT on the initial shock strength. Both quantities decrease rapidly with increasing $M_{s}$, however even in the case of the weakest constant-volume explosion shock, the distance to DDT is close to $L_{C J}^{\mathrm{min}}$. Therefore, $L_{C J}^{\mathrm{min}}$ can be used to estimate the minimal flame region required for the tDDT process.

\section{Transition density in the Chandresekhar-mass SNIa model}

We use the theory developed and validated above to estimate conditions, at which the tDDT can occur in SNIa. We restrict our analysis to the classical Chandrasekhar-mass model.

In the $\mathrm{M}_{c h}$ scenario, the explosion starts when a thermonuclear flame is ignited near the WD center and propagates in the gravitational field of a WD $(31,32,74)$. This flame is subject to the Rayleigh-Taylor (RT) instability, which generates convective flows and turbulence on multiple scales. The turbulent energy is transferred from larger to smaller scales through a turbulent cascade. The resulting turbulent flame propagates at subsonic speeds, thus allowing the WD to expand. The density of the burning material changes both with the distance from the WD center and due to the WD expansion.

Previous large-scale 3D simulations of SNIa explosions resolve scales from the WD size $\sim 1000 \mathrm{~km}$ down to $\sim 10 \mathrm{~km}$ using a computational mesh size $\lesssim 1 \mathrm{~km}(32,74)$. These scales are larger than the characteristic scales of thermonuclear flames, which are between $\sim 10^{-4} \mathrm{~cm}$ and $\sim 10 \mathrm{~m}$ for ${ }^{12} \mathrm{C}$ burning (60). Therefore, large-scale simulations rely on subgrid models that describe the physics of flames on unresolved scales and provide the flame speed for scales close to the computational mesh size. These simulations do not produce shocks unless detonations are artificially triggered at some point, and do not resolve any shock generation phenomena on scales $\lesssim 10 \mathrm{~km}$. Fully resolving these scales in a 3D simulation of an exploding WD remains computationally prohibitive.

Using known properties of laminar thermonuclear flames in the $50 / 50{ }^{12} \mathrm{C} /{ }^{16} \mathrm{O}$ mixture, which represents a typical WD composition, we computed $L_{C J}^{\min }, L_{C J}, U_{l}^{\max }$, and $U_{C J}$ using Equations 6, 11, 8, and 10. These are shown in Fig. 8 as functions of density. Both $L_{C J}^{\min }$ and $U_{C J}$ depend only on the mixture properties, which vary with local density. $L_{C J}$ and $U_{l}^{\max }$ also depend on the turbulent integral scale $l$ and velocity $U_{l}$. They were calculated assuming $l=10 \mathrm{~km}$ and $U_{l}=100 \mathrm{~km} \mathrm{~s}^{-1}$, values found in large-scale calculations of the $\mathbf{M}_{c h}$ explosions $(51,52,75)$. The lack of fully developed turbulence on scales $>10 \mathrm{~km}$ greatly reduces flame packing on these scales.

Figure 8 shows that $L_{C J}^{\min }$ decreases below $l=10 \mathrm{~km}$ at densities $>10^{7} \mathrm{~g} \mathrm{~cm}^{-3}$, thus allowing the $\mathrm{CJ}$ conditions to arise in the flow (76). As the density increases, the minimum size of the critical flame region decreases rapidly. At $\rho \approx 3 \times 10^{7} \mathrm{~g} \mathrm{~cm}^{-3}$, turbulent integral velocity $U_{l}^{\max }$ at the scale of $10 \mathrm{~km}$ required to produce tight flame packing associated with

$L_{C J}^{\min }$ becomes larger than the reference $U_{l}=100 \mathrm{~km} \mathrm{~s}^{-1}$. Therefore, at higher densities, high turbulent intensities not observed in full-star SNIa simulations $(51,52,75)$ would be required 
to pack the flame sufficiently tightly to achieve $L_{C J}^{\min }$. CJ conditions can be created by the turbulent intensity of $100 \mathrm{~km} \mathrm{~s}^{-1}$, though they would correspond to a less tightly packed flame and a larger critical flame volume $L_{C J}$. Corresponding values of $L_{C J}$ for the reference $U_{l}=100$ $\mathrm{km} \mathrm{s}^{-1}$, that we denote $L_{C J}^{r e f}$, as well as the turbulent velocity $U_{C J}$ at that scale, are also shown in Fig. 8. After the critical flame volume reaches a minimum value at $\rho \approx 3 \times 10^{7} \mathrm{~g} \mathrm{~cm}^{-3}$, it starts growing again, becoming larger than $10 \mathrm{~km}$ at $\rho \approx 1.5 \times 10^{8} \mathrm{~g} \mathrm{~cm}^{-3}$.

This shows that tDDT cannot occur for densities below $\approx 10^{7} \mathrm{~g} \mathrm{~cm}^{-3}$ and above $\approx 10^{8}$ $\mathrm{g} \mathrm{cm}^{-3}$ as it would require critical flame volumes that we do not expect to be produced by the turbulence present during a SNIa explosion. A critical flame volume smaller than the integral scale does not mean that tDDT will occur, given the stochastic nature of the process. As the ratio $L_{C J} / l$ becomes smaller, the probability of the formation of $\mathrm{CJ}$ conditions within the turbulent flame increases because a smaller size of the critical region allows for many more possible realizations in a given flow volume of size $l$. Therefore, the maximum probability of detonation formation is at the density corresponding to the smallest value of $L_{C J} \approx 2 \times 10^{3} \mathrm{~cm}$, or $l / L_{C J}^{\min } \approx$ 500 , at $\rho_{D D T} \approx 3 \times 10^{7} \mathrm{~g} \mathrm{~cm}^{-3}$. At that density, the number of possible realizations of the CJ conditions in a volume of size $l$, which could form over one integral-scale eddy turnover time, $\tau_{l}$, is

$$
N_{D D T} \sim\left(\frac{l}{L_{C J}^{\min }}\right)^{3}\left(\frac{\tau_{l}}{\tau_{L_{C J}}}\right)=\left(\frac{l}{L_{C J}^{\min }}\right)^{11 / 3} \sim 10^{10} .
$$

Here $\tau_{L_{C J}}$ is the eddy turnover time on scale $L_{C J}$. In other words, the probability of the formation of a flame configuration satisfying CJ conditions would have to be less than $10^{-10}$ to prevent the onset of the pressure runaway at this density in a given region of size $l$. Because $l$ is approximately two orders of magnitude smaller than the size of the WD $\sim 1000 \mathrm{~km}$, a large number of such regions would exist during the explosion inside the WD at the relevant densities. This further increases the probability of the onset of the runaway, thus making tDDT in the $\mathrm{M}_{c h}$ model almost inevitable.

\section{Discussion and conclusions}

We presented a self-consistent theory of turbulence-induced shock generation and DDT, and showed that it is in agreement with experiments involving chemical flames and direct numerical simulations of thermonuclear deflagrations in degenerate plasmas at conditions present in the stellar interior during a SNIa explosion. The overall process, which triggers pressure runaway and results in the formation of a strong shock, qualitatively is not sensitive to the details of the equation-of-state, microphysical transport, or reaction kinetics. Such runaway process will occur once the flame speed exceeds the speed of a CJ deflagration. This theory showed that in a ${ }^{12} \mathrm{C} /{ }^{16} \mathrm{O}$ WD in the classical $M_{c h}$ explosion scenario, tDDT has a high probability of occurrence at densities in the range $10^{7}-10^{8} \mathrm{~g} \mathrm{~cm}^{-3}$ with the maximum probability at $\rho_{D D T} \approx 3 \times 10^{7}$ $\mathrm{g} \mathrm{cm}^{-3}$. This value is similar to the transition densities $(1.3-2.4) \times 10^{7} \mathrm{~g} \mathrm{~cm}^{-3}$ adopted in 
subgrid-scale models used in prior large-scale calculations of the $\mathbf{M}_{c h}$ explosion scenario (32), which are consistent with observations.

This analysis assumed turbulent conditions previously observed in large-scale calculations of SNIa, namely integral velocity $U_{l}=100 \mathrm{~km} \mathrm{~s}^{-1}$ at the scale of $l=10 \mathrm{~km}$. This is in contrast with prior theoretical models $(50-52,77,78)$, which inherently required the formation of distributed flames to create conditions for the spontaneous reaction wave mechanism of DDT, and thus necessitated higher turbulent intensities typically above $\sim 1000 \mathrm{~km} \mathrm{~s}^{-1}$, which we regard as implausible.

The multiple proposed explosion scenarios for normal, bright SNIa share a common aspect - detonation formation at some point in the explosion. In particular, in the context of the $M_{c h}$ scenario, our analysis suggests that DDT is almost inevitable. The high probability of DDT given by Equation 13, however, makes it difficult for the $M_{c h}$ model to explain the class of sub-luminous SNIa, which were previously suggested to arise from purely deflagration-driven explosions (25). Furthermore, the validity of the $M_{c h}$ model has been questioned due to the lack of identified non-degenerate companion stars surviving the explosion (79) or ejecta interaction with the companions stars $(80)$ in some SNIa (81), though observational evidence of such interaction has been found in other events (82). If $M_{c h}$ is not the dominant channel for normal bright SNIa, then it becomes unclear whether WDs can grow to the Chandrasekhar mass because once this mass limit is reached then both core ignition and subsequent DDT would be almost unavoidable.

Our derived DDT conditions, $L_{C J}^{\min }$ and $U_{C J}$ (Equations 7, 10, depend only on the laminar flame properties, which in turn depend on the composition of stellar material in the interior of a WD at different radii. This suggests that the transition density $\rho_{D D T}$ could vary substantially between $50 / 50{ }^{12} \mathrm{C} /{ }^{16} \mathrm{O}$ mixtures and more ${ }^{12} \mathrm{C}$-poor compositions. Because the change in $\rho_{D D T}$ would result in a different total ${ }^{56} \mathrm{Ni}$ yield and thus luminosity, we predict a connection in the $M_{c h}$ scenario between the WD age and metallicity, which determine the interior composition, and the resulting SNIa light curve and spectral properties. This is potentially testable with observations. There may exist a minimal ${ }^{12} \mathrm{C}$ mass fraction, below which the onset of DDT would become unlikely due to either the high transition density, which would result in SNIa properties in disagreement with observations, or turbulent conditions required to produce superCJ turbulent flames, which are not observed in full-star SNIa simulations. Studies on the effect of mixture composition and metallicity on a SNIa explosion $(52,83,84)$ have suggested the dependence of $\rho_{D D T}$ on the ${ }^{12} \mathrm{C}$ mass fraction (52). However, those results were obtained for a fundamentally different DDT mechanism, which relies on the formation of spontaneous reaction waves in a reactivity gradient produced in a distributed flame, and an ad hoc prescription of $\rho_{D D T}(52,83,84)$. 


\section{Acknowledgments}

We thank the late A.M. Khokhlov (University of Chicago) for inspiring the work on this problem. AYP and VG also thank J.C. Wheeler (University of Texas, Austin) and E.S. Oran (Texas A\&M University) for valuable discussions. Rendering of Figure 5 as well as movies S1 and S2 was carried out by the Department of Defense (DoD) High Performance Computing Modernization Program (HPCMP) Data Analysis and Assessment Center (DAAC).

Funding: AYP and BDT were supported by the National Aeronautics and Space Administration (NASA) award NNH12AT33I. AYP was also supported by the Air Force Office of Scientific Research (AFOSR) award F4FGA06055G001. JC and KA were supported by the AFOSR award FA95501610403. VG was supported by the Alpha Foundation for the Improvement of Mine Safety and Health, Inc. through Grant No. AFC215FO-73. Computing resources were provided by the DoD HPCMP under the Frontier project award, and by the Naval Research Laboratory.

Author contributions: AYP conceived and oversaw the project, developed the theory and the code ATHENA-RFX, carried out DNS calculations and their analysis, applied the theory to SNIa, and led the writing of the manuscript. JC and KA developed the experimental facility and carried out experiments. VG developed the concept of shock amplification to a detonation through shock-flame interactions, contributed to the development of the overall theory and analysis of the DNS data, application of the theory to SNIa, and carried out simulations of shock-flame interactions. VG and KA contributed to the manuscript writing. BDT implemented thermonuclear reaction kinetics and transport in ATHENA-RFX.

Competing interests: The authors have no competing interests.

Data and materials availability: Experimental data for Figures $2 \mathrm{~A}, \mathrm{~B} \& \mathrm{4F}-\mathrm{J}$ are available in Data S1. Distribution of the ATHENA-RFX software falls under legal restrictions of the US government's International Traffic in Arms Regulation (ITAR) and Export Administration Regulation (EAR). Access to the code may require an export license from the Directorate of Defense Trade Controls, US Department of State. Subject to that provision, readers may obtain a copy of the software by contacting AYP. Output data for all the simulations, totaling 35 terabytes, is permanently archived at the Department of Defense (DoD) Supercomputing Resource Center (DSRC) at the Engineer Research and Development Center (ERDC) in Vicksburg, Mississippi. Transfer of this large data volume can be arranged by contacting AYP.

\section{Supplementary Materials}

Materials and Methods

Figures S1-S2

Table S1

Movies S1-S2

Data $\mathrm{S} 1$

References (85-95) 


\section{References}

1. M. M. Phillips. The absolute magnitudes of type Ia supernovae. Astrophys. J. 413:L105 (1993).

2. A.G. Riess, W.H. Press, R.P. Kirshner. Using type Ia supernova light curve shapes to measure the Hubble constant. Astrophys. J. 438:L17 (1995).

3. S. Jha, A. G. Riess, R. P. Kirshner. Improved distances to type Ia supernovae with multicolor light-curve shapes: MLCS2k2. Astrophys. J. 659:122 (2007).

4. F. Hoyle, W. A. Fowler. Nucleosynthesis in supernovae. ApJ, 132:565-590 (1960).

5. B. Wang, Z. Han. Progenitors of type Ia supernovae. New Astron. Rev. 56:122-141 (2012).

6. W. Hillebrandt, M. Kromer, F. K. Röpke, A. J. Ruiter. Towards an understanding of Type Ia supernovae from a synthesis of theory and observations. Front. Phys. 8:116-143 (2013).

7. D. Maoz, F. Mannucci, G. Nelemans. Observational clues to the progenitors of type Ia supernovae. Annu. Rev. Astron. Astrophys. 52:107-170 (2014).

8. F. K. Röpke, S. A. Sim. Models for type Ia supernovae and related astrophysical transients. Space Sci. Rev. 214:72 (2018)

9. A. M. Khokhlov. Delayed detonation model for type Ia supernovae. Astron. Astrophys. 245:114-128 (1991).

10. P. A. Hoflich, A. M. Khokhlov, J. C. Wheeler. Delayed detonation models for normal and subluminous type Ia supernovae:Absolute brightness, light curves, and molecule formation. Astrophys. J. 444:831-847 (1995).

11. V. N. Gamezo, A. M. Khokhlov, E. S. Oran, A. Y. Chtchelkanova, and R. O. Rosenberg. Thermonuclear supernovae: Simulations of the deflagration stage and their implications. Science, 299:77-81 (2003).

12. I. R. Seitenzahl, F. Ciaraldi-Schoolmann, F. K. Röpke, M. Fink, W. Hillebrandt, M. Kromer, R. Pakmor, A. J. Ruiter, S. A. Sim, S. Taubenberger. Three-dimensional delayeddetonation models with nucleosynthesis for Type Ia supernovae. Mon. Not. Royal Astron. Soc. 429:1156-1172 (2013).

13. S.E. Woosley, T.A. Weaver. Sub-Chandrasekhar mass models for type Ia supernovae. Astrophys. J. 423:371-379 (1994).

14. P. A. Hoflich, A. M. Khokhlov. Explosion models for type Ia supernovae: A comparison with observed light curves, distances, $H_{0}$, and $q_{0}$. Astrophys. J. 457:500-528 (1996). 
15. A. J. Ruiter, K. Belczynski, S. A.Sim, W. Hillebrandt, C. L. Fryer, M. Fink, M. Kromer. Delay times and rates for type Ia supernovae and thermonuclear explosions from doubledetonation sub-Chandrasekhar mass models. Mon. Not. Royal Astron. Soc. 417:408-419 (2011).

16. D. A. Goldstein, D. Kasen. Evidence for sub-Chandrasekhar mass type Ia supernovae from an extensive survey of radiative transfer models. Astrophys. J. 852:L33 (2018).

17. R. Mochkovitch, M. Livio. The coalescence of white dwarfs and type Ia supernovae. Astron. Astrophys. 209:111-118 (1989).

18. J. Guillochon, M. Dan, E. Ramirez-Ruiz, S. Rosswog. Prelude to a double degenerate merger: The onset of mass transfer and its impact on gravitational waves and surface detonations. Astrophys. J. 709:L64 (2010).

19. R. Pakmor, M. Kromer, F. K. Röpke, S. A. Sim, A. J. Ruiter, W. Hillebrandt. Sub-luminous type Ia supernovae from the mergers of equal-mass white dwarfs with mass $\sim 0.9 \mathrm{M}$. Nature 463:61 (2010).

20. O. Papish, H. B. Perets. Supernovae from direct collisions of white dwarfs and the role of helium shell ignition. Astrophys. J. 822:19 (2016).

21. T. Plewa, A. C. Calder, D. Q. Lamb. Type Ia supernova explosion: Gravitationally confined detonation. Astrophys. J. 612:L37-L40 (2004).

22. I. R. Seitenzahl, M. Kromer, S. T. Ohlmann, F. Ciaraldi-Schoolmann, K. Marquardt, Mi. Fink, W. Hillebrandt, R. Pakmor, F. K. Röpke, A. J. Ruiter, S. A. Sim, S. Taubenberger. Three-dimensional simulations of gravitationally confined detonations compared to observations of SN 1991T. Astron. Astrophys. 592:A57 (2016).

23. G. H. Marion et al. A Catalog of Near-infrared Spectra from Type Ia Supernovae. Astron. J. 138:727-757 (2009).

24. J. Parrent, B. Friesen, M. Parthasarathy. A review of type Ia supernova spectra. Astrophys. Space. Sci. 351:1-52 (2014).

25. M. Fink, M. Kromer, I. R. Seitenzahl, F. Ciaraldi-Schoolmann, F. K. Röpke, S. A. Sim, R. Pakmor, A. J. Ruiter, W. Hillebrandt Three-dimensional pure deflagration models with nucleosynthesis and synthetic observables for Type Ia supernovae. Mon. Not. Royal Astron. Soc. 438:1762-1783 (2014).

26. J. W. Meyer, A. K. Oppenheim. On the shock-induced ignition of explosive gases. Proc. Comb. Inst. 13:1153-1164 (1971). 
27. Ya. B. Zeldovich, V. B. Librovich, G. M. Makhviladze, G. I. Sivashinsky. Development of detonation in a nonuniformly preheated gas. Astron. Acta 15:313-321 (1970).

28. J. H. S. Lee, R. Knustautas, N. Yoshikawa. Photochemical initiation of gaseous detonations. Astron. Acta 5:971-982 (1978).

29. E. S. Oran, V. N. Gamezo. Origins of the deflagration-to-detonation transition in gas-phase combustion. Combust. Flame 148:4-47 (2007).

30. C. Raskin, E. Scannapieco, G. Rockefeller, C. Fryer, S. Diehl, F. X. Timmes. 56Ni production in double-degenerate white dwarf collisions. Astrophys. J. 724:111-125 (2010).

31. V. N. Gamezo, A. M. Khokhlov, E. S. Oran. Three-dimensional delayed-detonation model of type Ia supernova. Astrophys. J., 623:337-346 (2005).

32. F. K. Röpke, J. C. Niemeyer. Delayed detonations in full-star models of type Ia supernova explosions. Astron. Astrophys. 464:683-686 (2007).

33. K. Kailasanath. Review of propulsion applications of detonation waves. Amer. Inst. Aeronaut. Astronaut. J. 38:1698-1708 (2000).

34. K. Kailasanath. Recent developments in the research on pulse detonation engines. Amer. Inst. Aeronaut. Astronaut. J. 41:145-159 (2003).

35. P. Wolański. Detonative propulsion. Proc. Combust. Inst. 34:125-158 (2013).

36. F. K. Lu, E. M. Braun. Rotating detonation wave propulsion: Experimental challenges, modeling, and engine concepts. J. Prop. Power 30:1125-1142 (2014).

37. B. A. Rankin, M. L. Fotia, A. G. Naples, C. A. Stevens, J. L. Hoke, T. A. Kaemming, S. W. Theuerkauf, F. R. Schauer. Overview of performance, application, and analysis of rotating detonation engine technologies. J. Prop. Power 33:131-143 (2017).

38. R. K. Zipf, V. N. Gamezo, K. M. Mohamed, E. S. Oran, D. A. Kessler. Deflagration-todetonation transition in natural gas-air mixtures. Combust. Flame 161:2165-2176 (2014).

39. V. H. Y. Tam, D. M. Johnson. Detonation: should it be included in hazard and risk assessment? Chem. Eng. Trans. 48:451-456 (2016).

40. D. M. Johnson, V. H. Y. Tam. Why DDT is the only way to explain some vapor cloud explosions? Proc. Safety Prog. 36:292-300 (2017).

41. W. Luangdilok. The probability of DDT during severe accidents. Technical Bulletin N-1601, Fauske \& Associates, 2017. 
42. V. H. Y. Tam. The Buncefield accident - Why was the explosion so severe? Loss Prevention Bulletin, 222:2-8 (2011).

43. J. Taveau. The Buncefield explosion: Were the resulting overpressures really unforeseeable? Proc. Safety Prog. 31:55-71 (2012).

44. G. W. McMahon, J. R. Britt, J. L. O’Daniel, L. K. Davis, R. E. Walker. CFD study and structural analysis of the Sago mine accident. US Army Corps of Engineers, Engineer Research and Development Center (ERDC), Geotechnical and Structures Laboratory (GSL), 2007, ERDC/GSL TR-06-X, 138 pp.

45. Johnson, D.M. Characteristics of the vapour cloud explosion incident at the IOC terminal in Jaipur, 29th October 2009. 8th Global Congress on Process Safety, Houston, TX, 2012.

46. Report of the Japanese government to the IAEA ministerial conference on nuclear safety The accident at TEPCO's Fukushima nuclear power station, Nuclear Emergency Response Headquarters, Government of Japan (2011) https:// japan.kantei.go.jp/kan/ topics/201106/iaea_houkokusho_e.html.

47. A. Y. Poludnenko, T.A. Gardiner, E.S. Oran. Spontaneous transition of turbulent flames to detonations in unconfined media. Phys. Rev. Lett. 107:054501 (2011).

48. F. A. Williams, Combustion theory. 2nd edition, 1985, Benjamin/Cummings.

49. A. Y. Poludnenko. Pulsating instability and self-acceleration of fast turbulent flames. Phys. Fluids 27:014106 (2015).

50. A. M. Khokhlov, E. S. Oran, J. C. Wheeler. Deflagration-to-detonation transition in thermonuclear supernovae. Astrophys. J. 478:678-688 (1997b).

51. J. C. Niemeyer, S. E. Woosley. The thermonuclear explosion of Chandrasekhar mass white dwarfs. Astrophys. J. 475:740-753 (1997).

52. S. E. Woosley. Type Ia supernovae: Burning and detonation in the distributed regime. Astrophys. J. 668:1109-1117 (2007).

53. F. Ciaraldi-Schoolmann, I.R. Seitenzahl, F. K. Röpke. A subgrid-scale model for deflagration-to-detonation transitions in Type Ia supernova explosion simulations. Numerical implementation. Astron. Astrophys. 559:A117 (2013).

54. V.N. Gamezo, A.Y. Poludnenko, E.S. Oran. One-dimensional evolution of fast flames. Proceedings of the $23^{\text {rd }}$ International Colloquium on the Dynamics of Explosions and Reactive Systems, Irvine, CA, 25 July, 2011, paper 330.

55. J. F. Driscoll. Turbulent premixed combustion: Flamelet structure and its effect on turbulent burning velocities. Prog. Ener. Combust. Sci. 34:91-134 (2008). 
56. L. J. Dursi. The response of model and astrophysical thermonuclear flames to curvature and stretch. Astrophys. J. 595:955-979 (2003).

57. P. E. Hamlington, A. Y. Poludnenko, E. S. Oran. Interactions between turbulence and flames in premixed reacting flows. Phys. Fluids 23:125111 (2011).

58. P. E. Hamlington, A. Y. Poludnenko, E. S. Oran. Intermittency in premixed turbulent reacting flows. Phys. Fluids 24:075111 (2012).

59. C. A. Z. Towery, A. Y. Poludnenko, J. Urzay, J. O’Brien, M. Ihme, P. E. Hamlington. Spectral kinetic energy transfer in turbulent premixed reacting flows. Phys. Rev. E 93:053115 (2016).

60. F. X. Timmes, S. E. Woosley. The conductive propagation of nuclear flames. I - Degenerate $\mathrm{C}+\mathrm{O}$ and $\mathrm{O}+\mathrm{NE}+\mathrm{MG}$ white dwarfs. Astrophys. J. 396:649 (1992).

61. C. Charignon, J.-P. Chiéze. Deflagration-to-detonation transition by amplification of acoustic waves in type Ia supernovae. Astron. Astrophys. 550:A105 (2013).

62. Materials and methods are available as supplementary materials.

63. J. P. McGarry, K. A. Ahmed. Flame-turbulence interaction of laminar premixed deflagrated flames. Combust. Flame 176:439-450 (2017).

64. J. Chambers, K. Ahmed. Turbulent flame augmentation using a fluidic jet for deflagrationto-detonation. Fuel 199:616-626 (2017).

65. J. Sosa, J. Chambers, K. A. Ahmed, A. Poludnenko, V. N. Gamezo. Compressible turbulent flame speeds of highly turbulent standing flames. Proc. Combust. Inst. 37:3495-3502 (2019).

66. D.A. Kessler, V.N. Gamezo, E.S. Oran. Simulations of flame acceleration and deflagrationto-detonation transitions in methaneair systems. Combust. Flame 157:2063-2077 (2010).

67. J. M. Stone et al. Athena: A new code for astrophysical MHD. Astrophys. J. Supp. 178:137177 (2008).

68. A. Y. Poludnenko, E. S. Oran. The interaction of high-speed turbulence with flames: Global properties and internal flame structure. Combust. Flame 157:995-1011 (2010).

69. A. J. Aspden, J. B. Bell, M. S. Day, S. E. Woosley, M. Zingale. Turbulence-flame interactions in Type Ia supernovae. Astrophys. J. 689:1173-1185 (2008).

70. A. J. Aspden, J. B. Bell, S. E. Woosley. Distributed flames in Type Ia supernovae. Astrophys. J. 710:1654-1663 (2010). 
71. J.B. Bell, M.S. Day, C.A. Rendleman, S.E. Woosley, M. Zingale. Direct numerical simulations of type Ia supernovae flames. I. The Landau-Darrieus instability. Astrophys. J. 606:1029-1038 (2004).

72. J.B. Bell, M.S. Day, C.A. Rendleman, S.E. Woosley, M. Zingale. Direct numerical simulations of type Ia supernovae flames. II. The Rayleigh-Taylor instability. Astrophys. J. 608:883-906 (2004).

73. V.N. Gamezo, J.C. Wheeler, A.M. Khokhlov, E.S. Oran. Multilevel structure of cellular detonations in Type Ia supernovae. Astrophys. J. 512:827-842 (1999).

74. H. Ma, S. E. Woosley, C. M. Malone, A. Almgren, and J. Bell. Carbon deflagration in type Ia supernova. I. Centrally ignited models. Astrophys. J., 771:58 (2013).

75. F. K. Röpke. Flame-driven deflagration-to-detonation transitions in type Ia supernovae? Astrophys. J. 668:1103-1108 (2007).

76. At low densities $\rho \lesssim 10^{7} \mathrm{~g} \mathrm{~cm}^{-3}$, turbulence would break the flame causing it to enter the distributed burning regime $(69,70)$. The formation of a distributed flame does not preclude the possibility of tDDT via our tDDT mechanism provided that the burning speed of a distributed flame satisfies Equation 11. However, the expressions for critical turbulent conditions we derive are not applicable in that regime.

77. A. M. Khokhlov, E. S. Oran, J. C. Wheeler. A theory of deflagration-to-detonation transition in unconfined flames. Combust. Flame 108:503-517 (1997a).

78. S. E. Woosley, A. R. Kerstein, V. Sankaran, A. J. Aspden, F. K. Röpke. Type Ia supernovae: Calculations of turbulent flames using the linear eddy model. Astrophys. J. 704:255-273 (2009).

79. B.E. Schaefer, A. Pagnotta. An absence of ex-companion stars in the type Ia supernova remnant SNR 0509267.5 Nature 481:164-166 (2012).

80. R.P. Olling et al.No signature of ejecta interaction with a stellar companion in three type Ia supernovae. Nature 521:332-335 (2015).

81. B. J. Shappee, A. L. Piro , K. Z. Stanek, S. G. Patel, R. A. Margutti, V. M. Lipunov, R. W. Pogge. Strong evidence against a non-degenerate companion in SN 2012cg. Astrophys. J 855:6 (2018).

82. Y. Cao et al.A strong ultraviolet pulse from a newborn type Ia supernova. Nature 521:328331 (2015).

83. A. P. Jackson, A. C. Calder, D. M. Townsley, D. A. Chamulak, E. F. Brown, F. X. Timmes. Evaluating systematic dependencies of type Ia supernovae: The influence of deflagration to detonation density. Astrophys. J. 720:99-113 (2010). 
84. A. C. Calder, B. K. Krueger, A. P. Jackson, D. M. Townsley. The influence of chemical composition on models of Type Ia supernovae. Front. Phys. 8:168-188 (2013).

85. In principle, it could be possible to demonstrate this DDT process at lower densities and turbulent intensities using alternative modeling approaches, which do not aim to fully resolve the flame. In particular, by not resolving the flame, it could be possible to consider larger system sizes, which would be required for this DDT mechanism to operate at lower densities or in more ${ }^{12} \mathrm{C}$-poor mixtures. For instance, thickened flame techniques could represent one such possible strategy (86). It is important, however, for such modeling approaches to allow for the feedback process critical for this DDT mechanism to operate. In particular, they need to account for the changes in the local flame properties (speed and thickness) in response to the fuel compression during the runaway process, and the associated changes in the flame wrinkling by turbulence.

86. O. Colin, F. Ducros, D. Veynante, T. Poinsot. A thickened flame model for large eddy simulations of turbulent premixed combustion. Phys. Fluids. 12:1843-1863 (2000).

87. T. A. Gardiner, J. M. Stone. An unsplit Godunov method for ideal MHD via constrained transport in three dimensions. J. Comput. Phys. 227:4123-4141 (2008).

88. W. Hillebrandt, J. C. Niemeyer. Type Ia supernova explosion models. Annu. Rev. Astro. Astrophys. 38:191-230 (2000).

89. G. R. Caughlan, W. A. Fowler. Thermonuclear reaction rates V. At. Data Nucl. Data Tables 40:283 (1988).

90. F. X. Timmes, F. D. Swesty. The accuracy, consistency, and speed of an electron-positron equation of state based on table interpolation of the Helmholtz free energy. Astrophys. J. Supp. 126:501-516 (2000).

91. F. X. Timmes. Physical properties of laminar helium deflagrations. Astrophys. J. 528:913945 (2000).

92. F. Coquel, B. Perthame. Relaxation of energy and approximate Riemann solvers for general pressure laws in fluid dynamics. Soc. Industr. Appl. Math. J. Num. Anal. 35:2223-2249 (1998).

93. A. Khokhlov, I. Dominguez, C. Bacon, B. Clifford, E. Baron, P. Hoeflich, K. Krisciunas, N. Suntzeff, L. Wang. Three-dimensional simulations of thermonuclear detonation with $\alpha$-network: Numerical method and preliminary results. in Advances in computational astrophysics: Methods, tools, and outcome, R. Capuzzo-Dolcetta, M. Limongi, A. Tornambé, Eds. (Astron. Soc. Pacific Conf. Ser., 2012) vol. 453, pp. 107-114. 
94. N.N.Smirnov, V.B.Betelin, R.M.Shagaliev, V.F.Nikitin, I.M.Belyakov, Yu.N.Deryuguin, S.V.Aksenov, D.A.Korchazhkin. Hydrogen fuel rocket engines simulation using LOGOS code. Int. J. Hydr. Ener. 39:10748-10756 (2014).

95. N. N. Smirnov, V. B. Betelin, V. F. Nikitin, L. I. Stamov, D. I. Altoukhov. Accumulation of Errors in Numerical Simulations of Chemically Reacting Gas Dynamics. Acta Astronautica, 117:338-355 (2015) 

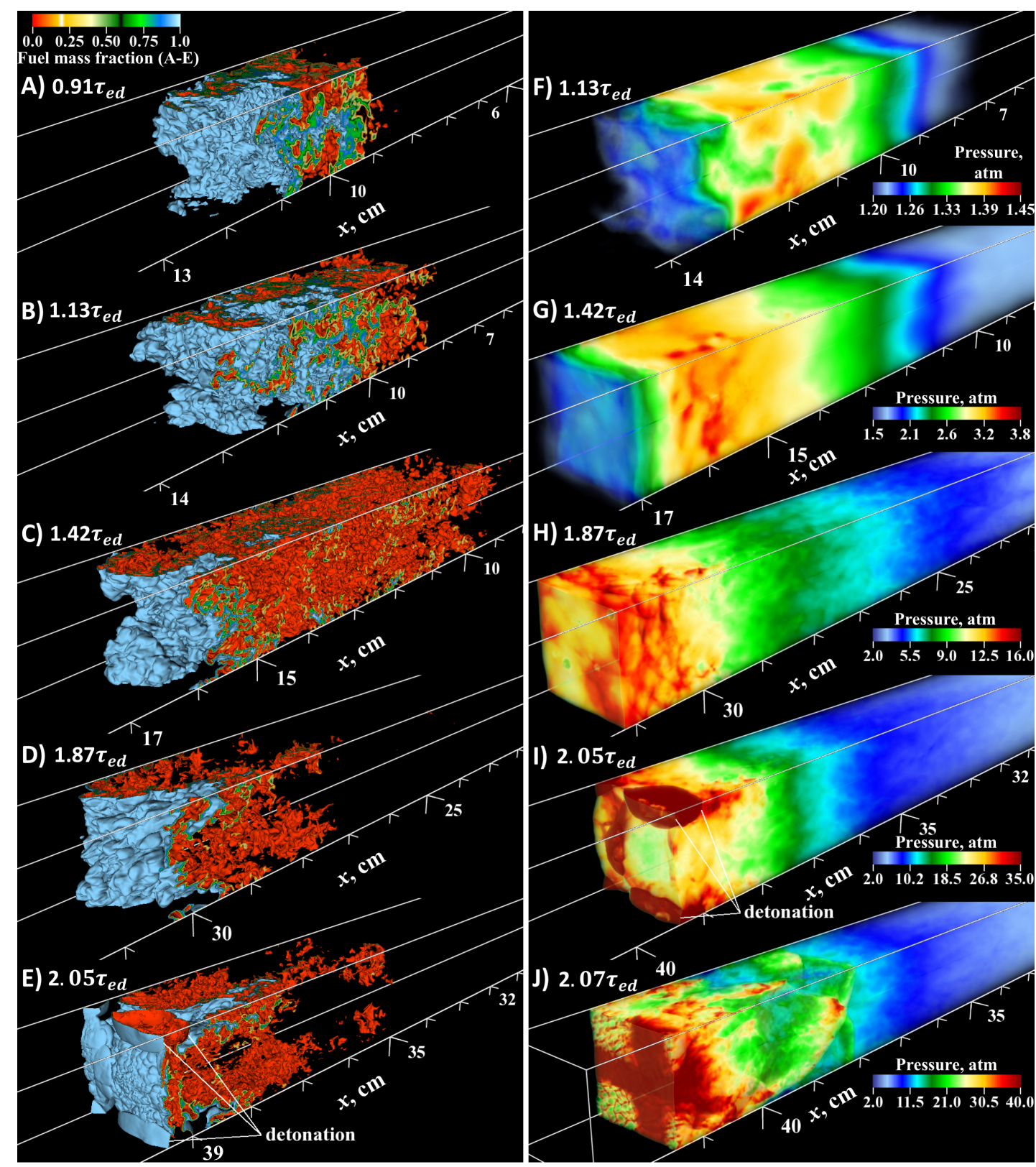

Figure 1: Evolution of a turbulent flame and transition to a detonation in a stoichiometric methane-air mixture (simulation reported as case 11 in Ref. (47)). Panels A - E: structure of a turbulent flame propagating to the left. Shown is the isovolume bounded by the two isosurfaces of the fuel mass fraction $Y=0.05$ and $Y=0.95$. Colors indicate the value of $Y$. Panels $F$ $J$ : corresponding pressure fields. Color scales in panels $\mathrm{F}$ - $\mathrm{J}$ show pressure normalized by the upstream pressure in the domain, $P_{0}=1 \mathrm{~atm}$, and the color scale changes between panels as the maximum pressure increases. Time for each frame is given in units of the eddy turnover time $\tau_{e d}=0.367 \mathrm{~ms}$. Corresponding turbulent flame speed and maximum pressures in the domain are shown in Fig.2. This simulation is shown in Movie S1. 

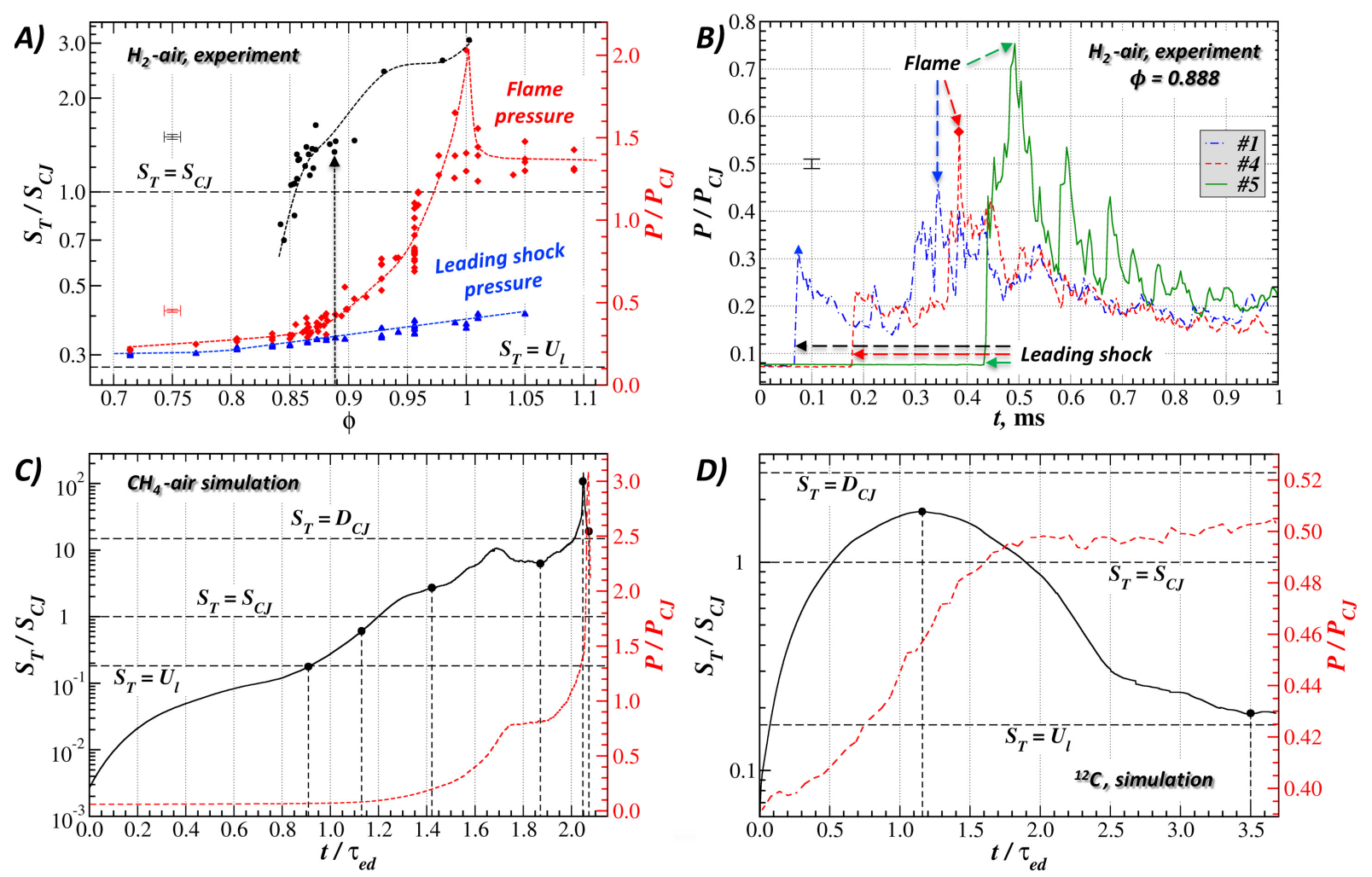

Figure 2: Pressures, $P$, and turbulent flame speeds, $S_{T}$, observed in experiments $(\mathbf{A}, \mathbf{B})$ and simulations (C,D) for chemical (A-C) and thermonuclear (D) flames. Panel A: summary of all experiments in hydrogen-air mixtures as a function of the equivalence ratio. Black circles: $S_{T}$ measured at the beginning of the first test section (Fig. 3). Values of $S_{T}$ are scaled by the CJ deflagration velocity $S_{C J}$, which depends on $\phi$ and varies between 192 and $292 \mathrm{~m} \mathrm{~s}^{-1}$. Blue triangles: peak pressures of the leading shock. Red diamonds: maximum pressures generated by the turbulent flame in the first test section. All pressures are scaled by the CJ detonation pressure $P_{C J}$, which depends on $\phi$ and varies between 14.0 and $15.8 \mathrm{~atm}$. Blue triangles and red diamonds in panel A correspond to pressure peaks indicated by a blue triangle and a red diamond in panel B, respectively. Black arrow marks the experiment shown in panels $\mathrm{F}-\mathrm{H}$ in Fig. 4. while the black circle immediately to the right marks the experiment shown in panels I and J. Panel B: pressure histories recorded in the experiment with $\phi=0.888$ by three pressure transducers indicated in Fig. 3 . Pressure is scaled by $P_{C J}=15.06$ atm. The first peak in each curve corresponds to the leading shock. The second pressure maximum is associated with the compressed region ahead of the flame (Fig. 4). Panel C: $S_{T}$ and maximum instantaneous $P$ in the computational domain as functions of time in the same simulation as Fig. 1. Initial pressure $P_{0}=1 \mathrm{~atm} . S_{T}$ is scaled by $S_{C J}=121.73 \mathrm{~m} \mathrm{~s}^{-1}, P$ is scaled by $P_{C J}=17.12$ atm, time is scaled by the eddy turnover time $\tau_{e d}=0.367 \mathrm{~ms}$. Vertical dashed lines indicate times corresponding to frames shown in Fig. 1. Panel $D: S_{T}$ and maximum instantaneous $P$ in the computational domain in the simulation of a thermonuclear turbulent flame in pure ${ }^{12} \mathrm{C}$ at initial density $\rho_{0}=4 \times 10^{8} \mathrm{~g} \mathrm{~cm}^{-3} . S_{T}$ is scaled by $S_{C J}=4.8 \times 10^{8} \mathrm{~cm} \mathrm{~s}^{-1}, P$ is scaled by $P_{C J}=3.64 \times 10^{26} \mathrm{ergs} \mathrm{cm}{ }^{-3}$, time is $6_{6}$ scaled by the integral-scale eddy turnover time $\tau_{e d}=5.76 \times 10^{-11} \mathrm{~s}$. Vertical dashed lines indicate times corresponding to frames shown in Fig. 5. Experimental uncertainties in quantities shown in panels $\mathrm{A}$ and $\mathrm{B}$ are indicated with error bars (62). 


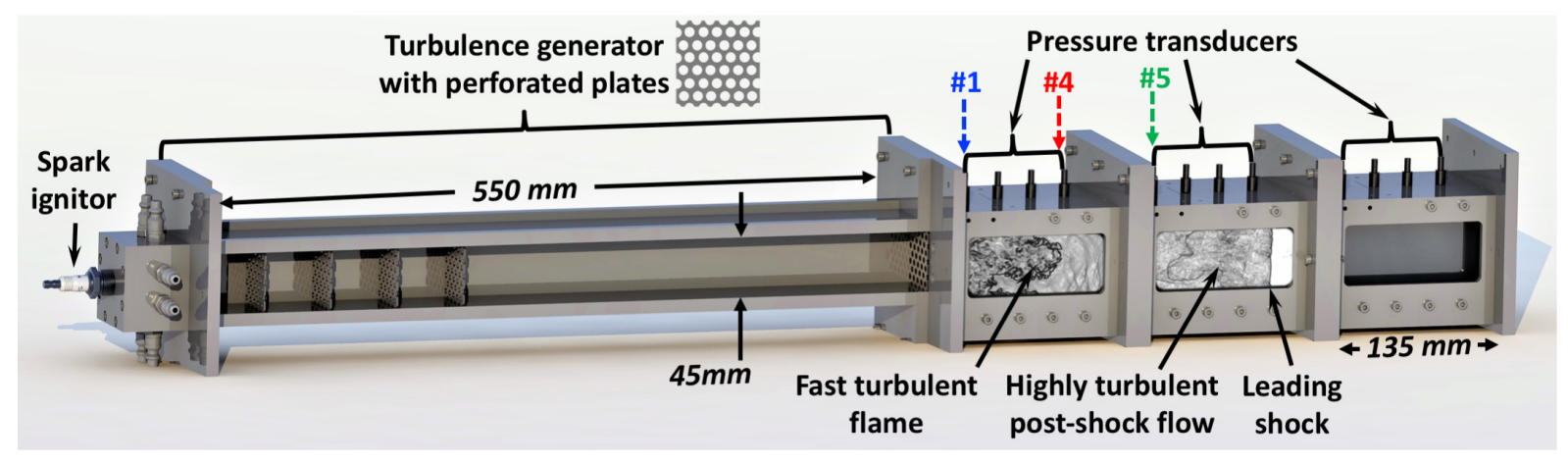

Figure 3: Experimental Turbulent Shock Tube (TST) facility. The section with perforated plates on the left is used to generate a fast turbulent flame, which propagates to the right. Test section is located past the last perforated plate at the distance of $550 \mathrm{~mm}$ from the ignition point. Blue, red, and green dashed arrows indicate the transducers \#1, \#4, and \#5 that produced pressure signals shown in Fig. $2 \mathrm{~B}$. 

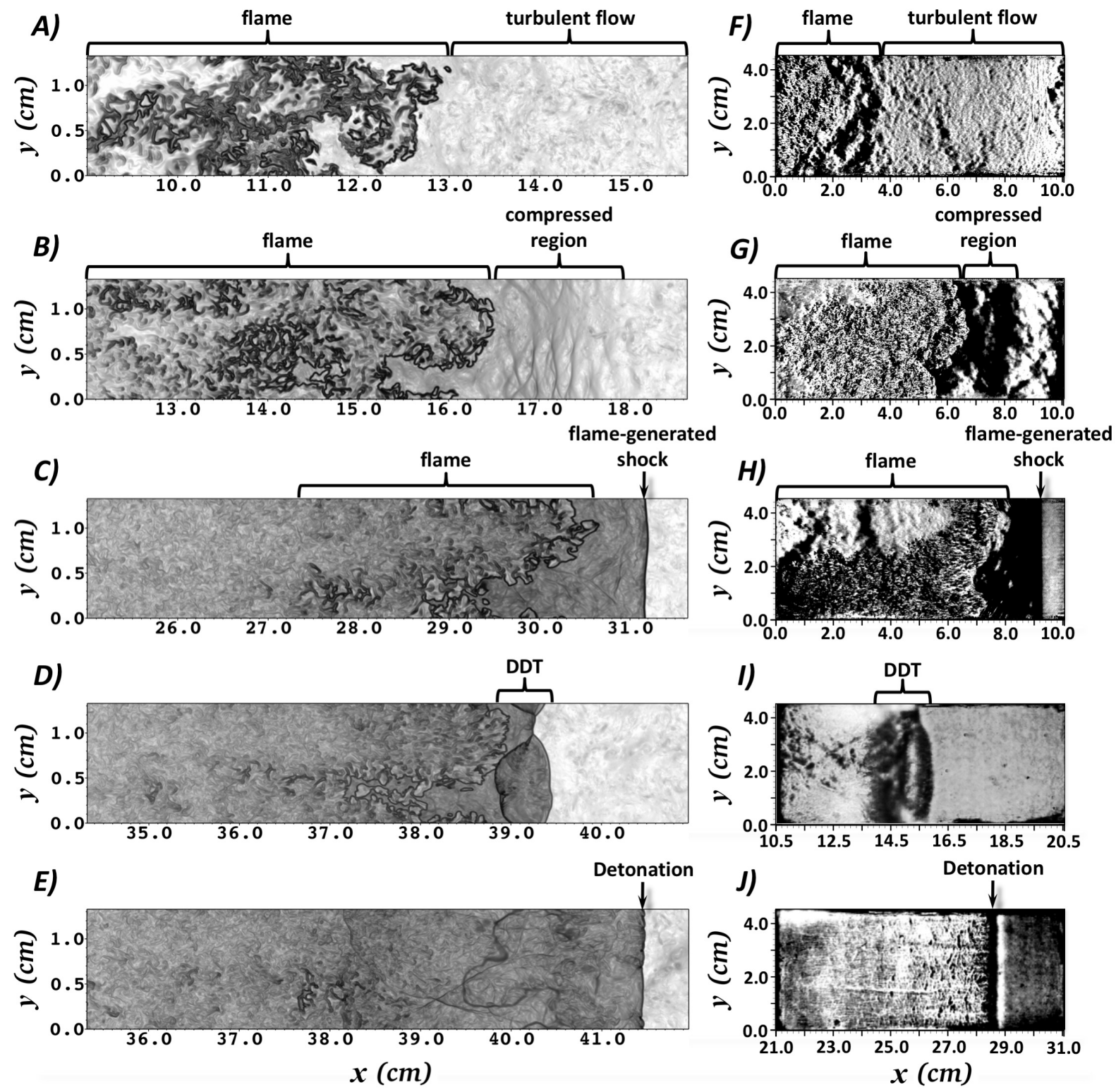

Figure 4: Simulated (A-E) and experimental (F-J) schlieren images of the turbulent flame during the pressure runaway and subsequent detonation formation. Experiment in panels F-H was carried out at $\phi=0.888$ (cf. Fig. 2B), experiment in panels I and J was carried out at $\phi=0.905$. Simulated schlieren images shown in panels A-E are from the same simulation as Fig. 1 . 


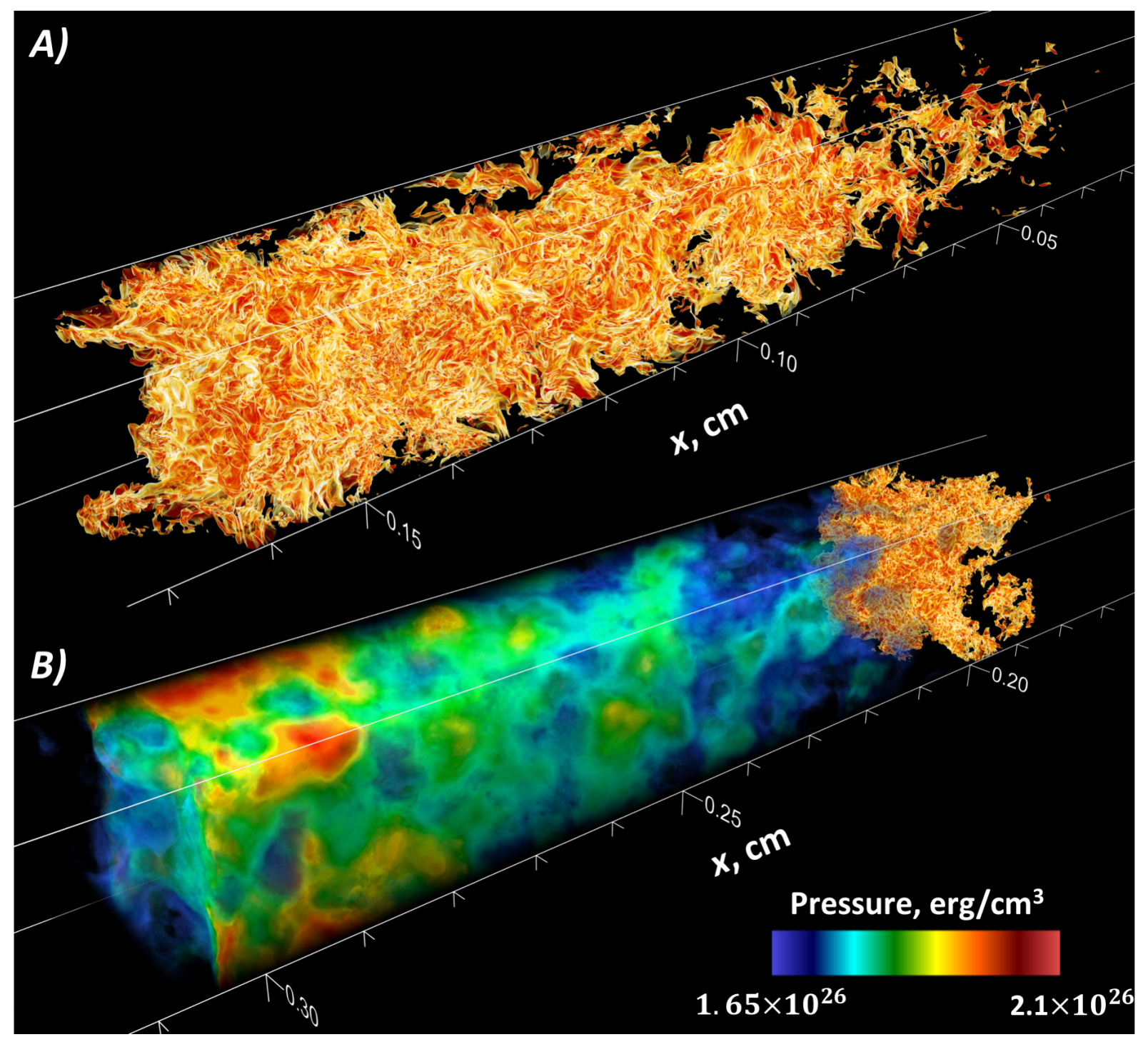

Figure 5: Turbulence-driven spontaneous shock formation in a turbulent thermonuclear flame. Panel A: flame structure at the time of maximum turbulent burning velocity, $S_{T}$. Panel $B$ : resulting flame-generated pressure field and the equilibrium structure of a turbulent flame at the end of the burn-out phase. The flame propagates to the left, and is shown as a semi-transparent flame surface with varying opacity using a ray-tracing visualization technique. Colors correspond to the light intensity, which increases from red to yellow to white. Corresponding turbulent flame speeds and maximum pressures are shown in Fig. 2D. This simulation is shown in Movie S2. 

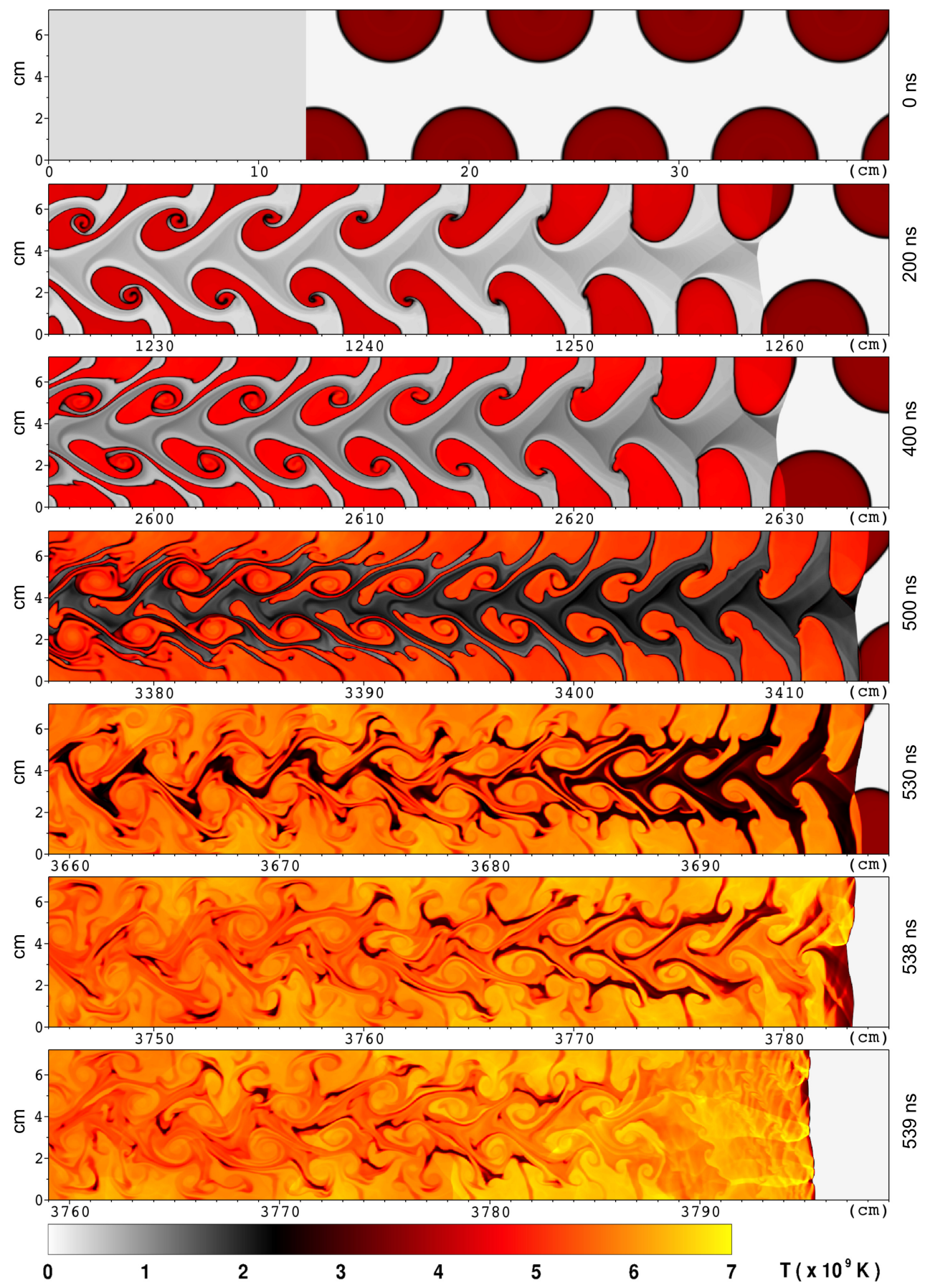

Figure 6: Shock interaction with an idealized turbulent thermonuclear flame and the resulting deflagration-to-detonation transition. Shown is the temperature distribution in a 2D calculation carried out at $\rho=3 \times 10^{7} \mathrm{~g} \mathrm{~cm}^{-3}$ in a $50 / 50{ }^{12} \mathrm{C}^{16} \mathrm{O}$ mixture. Initial shock Mach number is 1.28 corresponding to a constant-volume explosion. Time since the start of the simulation is indicated to the right of each frame. Detonation ignition occurs at $t=5.38 \times 10^{-7}$ s at $x \approx 3782 \mathrm{~cm}$. 

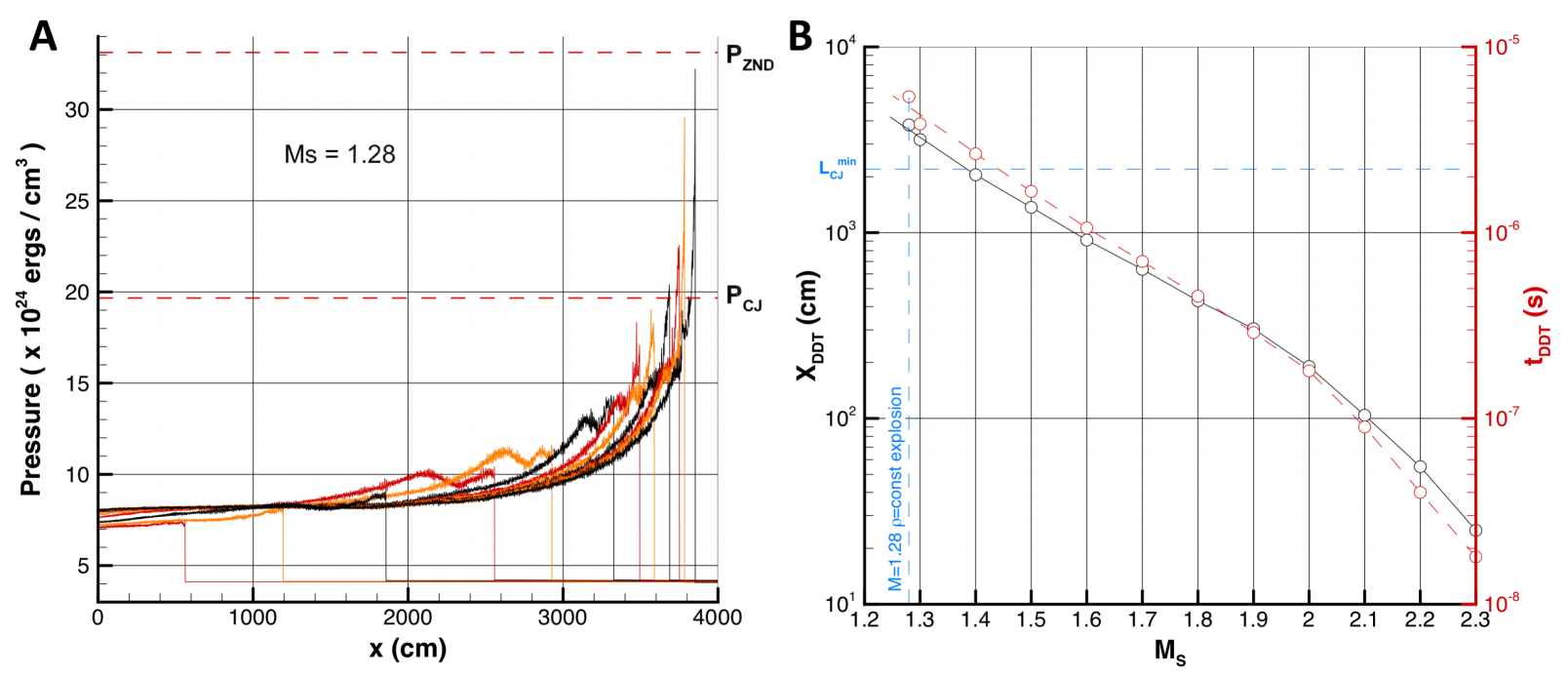

Figure 7: Deflagration-to-detonation transition driven by shock-flame interaction. Panel A: Pressure evolution in the calculation shown in Fig. 6. Shown is the $y$-averaged distribution of pressure in the domain for several time instances. Several colors are used to separate overlapping curves. Panel B: Distance (left axis) and time (right axis) to DDT (cf. Fig. 6) as a function of the shock Mach number. 


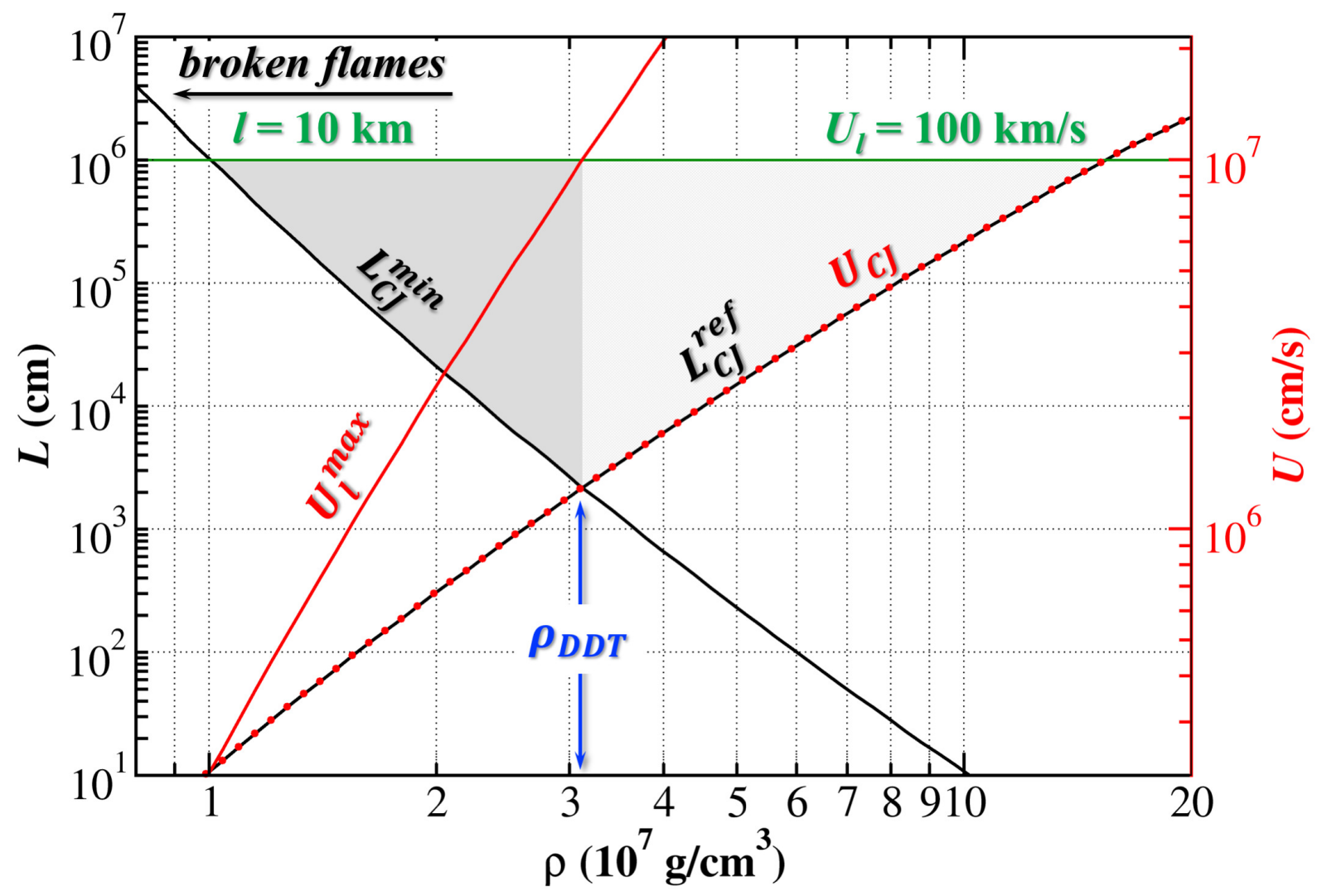

Figure 8: Critical conditions for tDDT in the Chandrasekhar-mass explosion scenario. Quantities shown are: $L_{C J}^{\min }$ (Equation 7), $U_{l}^{\max }$ (Equation 8), $L_{C J}^{r e f}$ (Equation 11), $U_{C J}$ (Equation 10). Gray areas show the range of parameters where tDDT is possible, with the highest probability corresponding to $\rho_{D D T}$. 


\title{
Science MIAAAS
}

\section{Supplementary Materials for}

A Unified Mechanism for Unconfined Deflagration-to-Detonation Transition in Terrestrial Chemical Systems and Type Ia Supernovae

\author{
Alexei Y. Poludnenko,* Jessica Chambers, Kareem Ahmed, \\ Vadim N. Gamezo, Brian D. Taylor \\ *Correspondence to: alexei.poludnenko@uconn.edu
}

This PDF file includes:

Materials and Methods

Supplementary Text

Figs. S1 and S2

Table S1

Captions for Movies S1 and S2

References (86-96)

Other Supplementary Materials for this manuscript include the following:

Movies S1 and S2

Data file S1 


\section{Materials and methods}

Here we provide further details of the experiments and numerical simulations, including the flow-field diagnostics, choice of thermonuclear plasma conditions in the 3D simulation of turbulence-flame interactions, and the characterization of the numerical simulation parameters and uncertainties.

\section{Experimental flow control equipment}

The TST facility operation begins with the opening of the fuel and air lines and setting the appropriate flow rates for the given conditions of interest. After the fill time of 20 seconds required to ensure the uniform volumetric fill, a transistor-transistor logic signal triggers the solenoid valve to halt the chamber filling and exhaust the incoming mixture. The mixture in the chamber is allowed to settle for 3 seconds, after which a $21 \mathrm{~ms}$ pulse signal is sent to the ignition coil. This powers the spark plug and a flame kernel ignites. At this point, signals are sent to initiate the procedure for optical and pressure diagnostics.

The control system, shown in Fig. S1, is designed to produce a homogeneous mixture of fuel and air at standard temperature and pressure within the facility before ignition. Premixed hydrogen and air are issued into the facility at low pressures and flow rates to reduce convective velocity and initial turbulence in the channel. We used main flow rates set to achieve the desired equivalence ratio in the range $\phi=0.78-1.1$. The air supplied by a compressor is regulated using an SMC Pneumatics \#AW20-N02E-CZ pressure regulator and then directed to a Dwyer VFA-6-BV flow meter. Hydrogen supplied by a high pressure tank is regulated using a Specialty

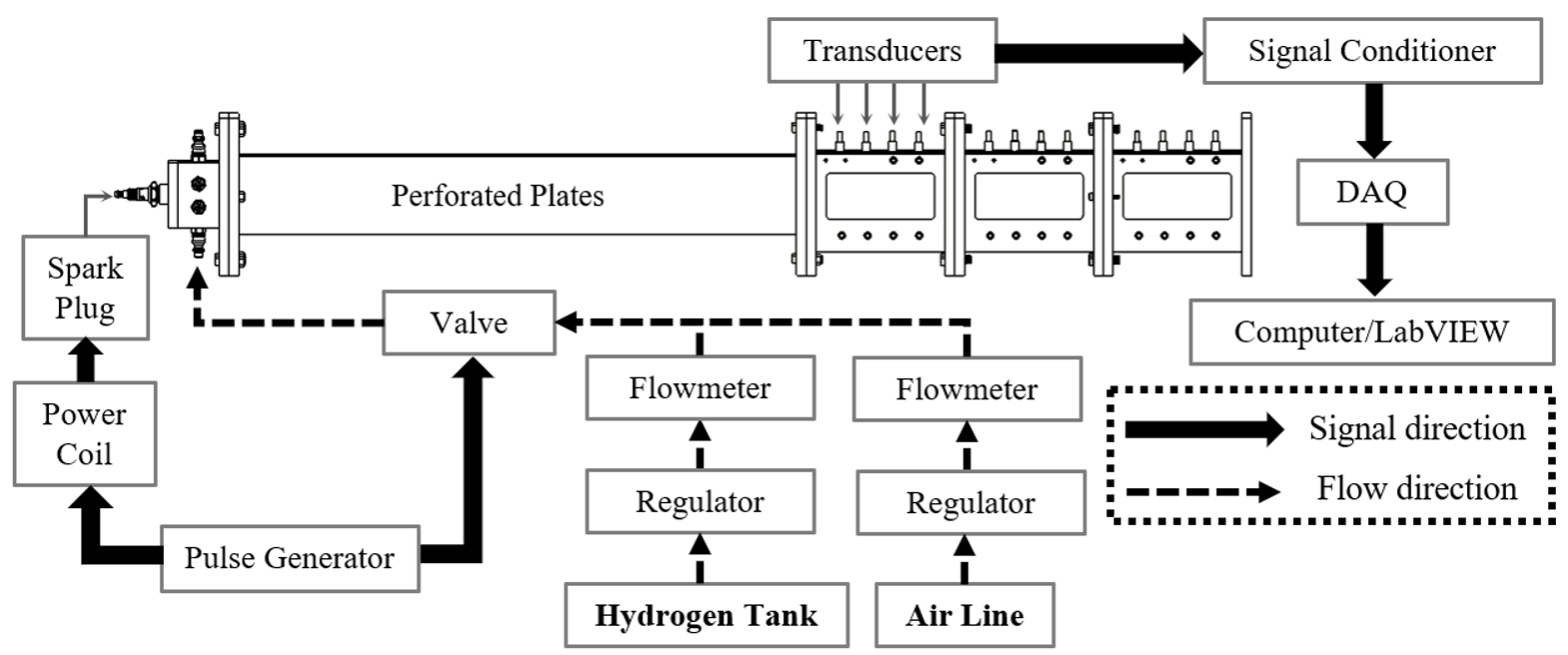

Figure S1: Experimental setup with flow and signal direction for the TST facility shown in Fig. 3. 
Gases Southeast Inc. \#HP-702-125-000-A regulator. It then flows to a VFA-3-BV flow meter for flow rate control. Desired equivalence ratios are achieved using flow rates in the range $28-30 \pm 0.9$ standard cubic feet per hour (SCFH) for air and $11-12.5 \pm 0.4 \mathrm{SCFH}$ for fuel. The fuel flow rate is corrected for use on the air-calibrated flow meter. The fuel and air lines merge to provide premixing in one line, which is then fed into the closed end of the facility. In order to prevent the flashback into the premixed line, a MAC 225B-111BAAA 3-way solenoid valve is used to divert the flow to an exhaust line immediately before ignition. A BNC Model 575 Pulse/Delay Generator is programmed with timing specifications to trigger the solenoid valve for exhaust, spark plug for ignition, and other equipment for diagnostics. The signal for the spark plug is routed to an ACCEL Super Coil 140001, which then powers the spark and immediately ignites the reactant mixture.

\section{Experimental diagnostics}

Schlieren diagnostics are used to probe the shock and flame behavior and to quantify their respective laboratory-frame velocities throughout the test-section domain before targeting specific conditions with particle image velocimetry (PIV). A standard Z arrangement schlieren is set up using two $152.5 \mathrm{~mm}$ diameter parabolic mirrors with a focal length of $1.525 \mathrm{~m}$. The images are captured using a Photron Fastcam SA-Z camera with the 1,024 $\times 1,024$ pixel spatial resolution, 16-bit range depth, and $100 \mathrm{kHz}$ recording rate. The camera is equipped with Nikon lens of focal length $200 \mathrm{~mm}$ and $f / 2.8$. The camera captures the entire window view and the resulting image resolution is $175 \mu \mathrm{m} \mathrm{px}^{-1}$ with a pixel-based velocity uncertainty of $5.6 \mathrm{~m} \mathrm{~s}^{-1}$.

Dynamic pressure transducers are lined within the test section to capture pressure evolution in the flow. Four PCB Model \#113B26 transducers are positioned with intervals of $25 \mathrm{~mm}$ in the axial direction along the spanwise centerline of the top plate of the test section (Fig. 3). Transducers have sensitivity of $10 \mathrm{mV} \mathrm{psi}^{-1}$ and are operated at a frequency of $250 \mathrm{kHz}$ to resolve pressure variations in time. Considering the non-linearity and sensitivity in the transducer response, the resolved static pressure values have the uncertainty of \pm 0.17 atm. Transducers are connected to a PCB signal conditioner 482C Series to amplify voltage signals and then are routed to a data acquisition device with the LabVIEW control hardware and software.

Once all conditions of interest are determined using schlieren and pressure measurements, high-speed PIV and $\mathrm{OH}^{*}$ chemiluminescence are used for the flow-field analysis. High-speed two-dimensional (2D) PIV is used to acquire the quantitative flow-field information for the characterization of turbulent conditions ahead of the flame. A Nd:YAG Lee LDP Dual Laser with a maximum power of $25 \mathrm{~mJ}$ is operated at $532 \mathrm{~nm}$. The laser beam travels through a set of optics, which converges the beam to a diameter $<1 \mathrm{~mm}$. The beam then reflects as a sheet up into the center of the test section to illuminate a $2 \mathrm{D}$ slice of the flow field. The main flow is seeded with $0.2 \mu \mathrm{m}$ aluminum oxide particles using a seeder designed with three levels of filtration for uniform seed density. The pulse separation of $2 \mu \mathrm{s}$ is intended to resolve the mean convective velocity of the shock, post-shock reactants, and the flame. The Photron Fastcam SA-Z camera equipped with a Nikon lens with focal length of $50 \mathrm{~mm}$ and $f / 1.2$ is 
used with a frame straddling method to achieve $20 \mathrm{kHz}$ PIV. Resulting PIV resolution is 42 $\mu \mathrm{m} \mathrm{px}^{-1}$ and measurement scale $\lambda_{m}=168 \mu \mathrm{m}$ with the field of view of $44 \times 22 \mathrm{~mm}$. The imaging maintained a ratio of the particle image diameter $d_{\tau}$ to pixel size $d_{p i x}$ at approximately $d_{\tau} / d_{p i x} \sim 1.5$. This results in the spatial resolution of $168 \mu \mathrm{m}$, which is approximately a half of the laminar flame thickness, and measurement scale relative to the approximate Kolmogorov scale $\lambda_{m} / \eta \sim 20-100$. DaVis software is used for processing the PIV images with the 30 step multi-pass method leading to a $16 \times 16$ pixel interrogation box and a $75 \%$ overlap, which results in the peak velocity uncertainty $<5 \mathrm{~m} \mathrm{~s}^{-1}$.

Simultaneous $\mathrm{OH}^{*}$ chemiluminescence is used to capture instantaneous images of the combustion product luminescence. The flame profile can then be outlined and superimposed onto the PIV images to identify the exact flame-flow conditions. A frame rate of $40 \mathrm{kHz}$ is achieved using another Photron Fastcam SA-Z camera. A Nikon lens with the focal length of $50 \mathrm{~mm}$ and $f / 1.2$ produces a resolution of $156 \mu \mathrm{m} \mathrm{px}^{-1}$ with the pixel-based velocity uncertainty of $3 \mathrm{~m} \mathrm{~s}^{-1}$. In post-processing, a gamma correction of 0.465 is used to brighten the images and reveal the flame-front structure. A calibration image is taken to coordinate both the PIV and chemiluminescence domains for the flame-front alignment.

\section{Experimental uncertainties}

The image sensor used for the flow imaging and diagnostics is a CMOS sensor with $1024 \times 1024$ pixels and $20 \times 20 \mu \mathrm{m}^{2}$ pixel size. The sensor sensitivity peak is at $680 \mathrm{~nm}$. The relative spectral response is $87 \%$ of peak value at $600 \mathrm{~nm}$ and $70 \%$ of peak value at $532 \mathrm{~nm}$. The viewing test section is optically accessible on three walls, which are recessed and fitted with fused silica quartz glass. The glass allows for transmission over $250-700 \mathrm{~nm}$ at $95 \%$ transmittance required for the laser diagnostics. Theoretical light transmittance in percentage, $\sigma$, of the optical system is calculated using

$$
\sigma_{\lambda}=100 \times \eta_{\text {glass }} \times s_{\lambda},
$$

where $\eta_{\text {glass }}$ is the relative amount of light transmitted by the optical component and $s_{\lambda}$ is the image sensor relative sensitivity at a given wavelength. This results in $82.65 \pm 4.1 \%$ transmittance at $600 \mathrm{~nm}$ and $66.5 \pm 3.4 \%$ transmittance at $532 \mathrm{~nm}$. The uncertainty was calculated using the manufacturer's reported uncertainty and the propagation of error approach.

DaVis software is used for processing the PIV images with a 30 step multi-pass method using a $16 \times 16$ pixel interrogation and a $75 \%$ overlap resulting in a peak velocity uncertainty

$<5 \mathrm{~m} \mathrm{~s}^{-1}$. The flame propagation velocity, $U_{f}$, is estimated using a forward differencing approach

$$
U_{f}=\frac{x_{f}(t+\Delta t)-x_{f}(t)}{\Delta t},
$$

where $x_{f}$ is the flame position.

Resulting uncertainties in all key experimentally measured quantities are shown in Fig. 2A,B and are as follows: 
- Pressure: \pm 0.17 atm, or $\pm 0.01 P / P_{C J}$.

- Velocity: $\pm 5 \mathrm{~m} \mathrm{~s}^{-1}$, or $0.026 S_{T} / S_{C J}$.

- Equivalence ratio: \pm 0.007 .

\section{Choice of fuel density and composition for the simulation of tDDT in ther- monuclear flames}

The choice of the mixture density and composition used in the numerical simulation of a turbulent thermonuclear deflagration was governed by the following considerations. The goal of this calculation is to demonstrate two points:

1. Interaction of a subsonic flame with turbulence, which is not only highly subsonic but also which has an integral velocity below $S_{C J}$, can produce turbulent flames with burning speeds far above $S_{C J}$.

2. When such high super-CJ burning velocities are reached, this results in the formation of strong shocks, capable of igniting a detonation.

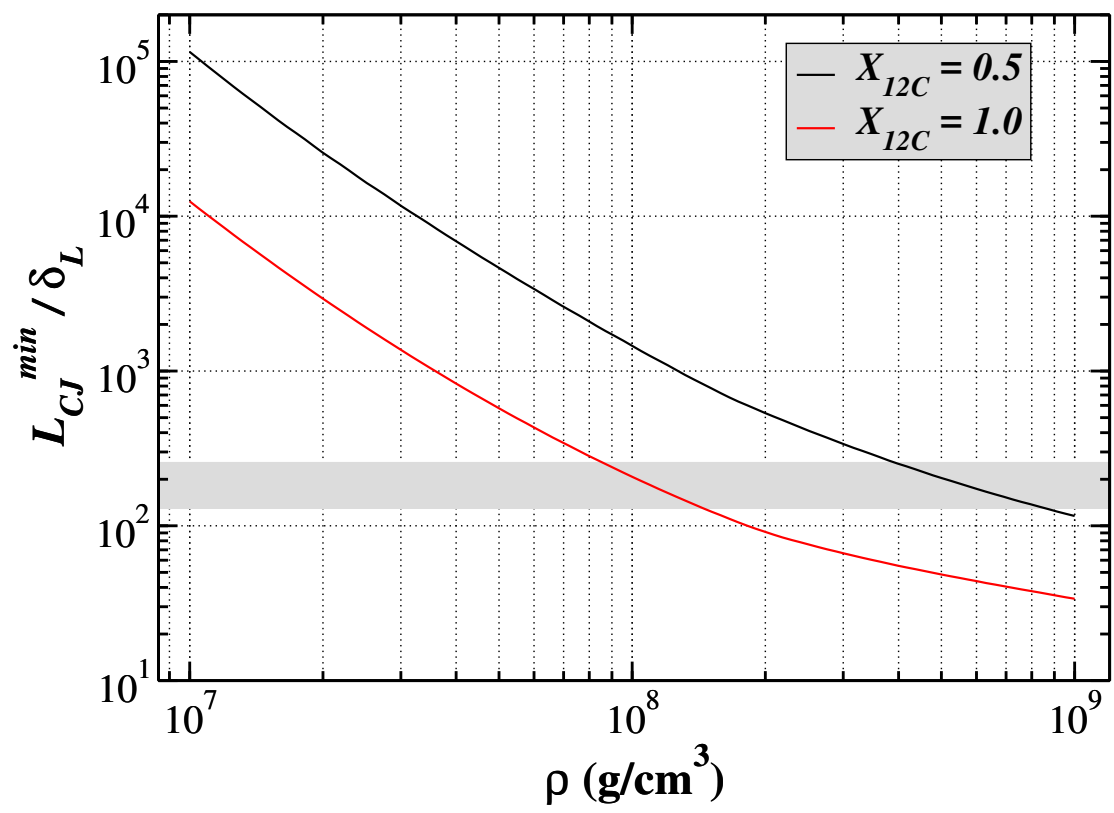

Figure S2: Minimum size of the flame region, in which tDDT can occur, $L_{C J}^{\min }$ (Eq. 7), normalized by the laminar flame width, $\delta_{L}$. Shaded gray region shows the range of scales, which can be captured in a simulation with the domain width of $512-1024$ cells and the resolution $\Delta x=\delta_{L} / 4$. 
As the first step, these two effects must be demonstrated in a first-principles 3D simulation, in which the laminar flame, i.e., ${ }^{12}$ C-burning zone, is fully resolved. Carrying out such a 3D simulation, however, faces the issue of computational feasibility.

We considered a variety of mixture compositions and densities in search of the conditions, at which the tDDT could be observed. Figure S2 shows the normalized minimum turbulent flame thickness required for the tDDT, $L_{C J}^{\min } / \delta_{L}$ (Eq. 7), as a function of density for two compositions: pure ${ }^{12} \mathrm{C}$ and $50 / 50$ mixture of ${ }^{12} \mathrm{C}$ and ${ }^{16} \mathrm{O}$. The latter composition would be more representative of the realistic white dwarf conditions, though even more ${ }^{12} \mathrm{C}$-poor mixtures could exist depending on the progenitor pre-explosion evolution.

In a simulation, resolution of at least 4 cells per $\delta_{L}$ would be required in order to capture the flame dynamics. Consequently, assuming this flame resolution and also assuming that the domain width in a simulation is equal to $L_{C J}^{\min }$ (which is the case in the presented calculation), Fig. S2 shows the range of scales, which can be captured in calculations with domain widths ranging from 512 to 1024 cells. The computational domain typically has to have an aspect ratio of at least $4: 1$ to $8: 1$ in the direction of flame propagation to accommodate the flame motion, though typically such aspect ratios must be much larger to allow a shock wave to develop ahead of the turbulent flame (it was 16: 1 in our final calculation). Therefore, this translates to the computational domain sizes of at least $512^{3} \times 4$ to $1024^{3} \times 16$, which are plausible with modern computational resources given the high computational cost of the equation of state, degenerate plasma transport, and reaction kinetics.

Figure S2 shows that demonstrating the tDDT process at densities close to $10^{7} \mathrm{~g} \mathrm{~cm}^{-3}$, at which DDT would be expected in a $M_{c h}$ explosion scenario, would require domain sizes far beyond what is feasible with current computational resources. Figure S2 also shows that in a realistic $50 / 50{ }^{12} \mathrm{C} /{ }^{16} \mathrm{O}$ mixture, $L_{C J}^{\min }$ could be captured in a 512-cell-wide computational domain, which was the domain size used in our calculation, only at densities approaching $10^{9}$

$\mathrm{g} \mathrm{cm}^{-3}$. At such high densities, however, required turbulent intensities based on Eqs. 8 or 10 become so large that fuel heating due to turbulent energy dissipation would result in excessive increase in temperature. This could cause spontaneous autoignition rendering the entire flame evolution irrelevant to the DDT process. At the same time, in a pure ${ }^{12} \mathrm{C}$ mixture, $L_{C J}^{\min }$ can be captured in a 512-cell-wide domain at densities close to $2 \times 10^{8} \mathrm{~g} \mathrm{~cm}^{-3}$ or above. Hence, we consider a pure ${ }^{12} \mathrm{C}$ mixture and a slightly higher density of $4 \times 10^{8} \mathrm{~g} \mathrm{~cm}^{-3}$.

With this choice of fuel composition and density, turbulent intensity required for the formation of a super-CJ flame was constrained based on the Eqs. 8 or 10. Although in a realistic SNIa, DDT would not be expected to occur at such turbulent intensity or in such a mixture, this choice of conditions allows one to demonstrate the two key points listed above using first-principles $3 \mathrm{D}$ simulations with a suitable equation of state, transport, and reaction kinetics (86).

\section{Numerical methods and uncertainties}

Turbulence-flame interactions for both chemical and thermonuclear flames are modeled using the code ATHENA-RFX $(68,69)$, which is the reactive flow extension of the magnetohydro- 
dynamic code Athena (68) (also see (88)). ATHENA-RFX is a fixed-grid, massively parallel, finite-volume, fully conservative code. It solves compressible reactive-flow equations, and implements a fully unsplit corner transport upwind scheme with the piecewise-parabolic (PPM) spatial reconstruction and the Harten-Lax-van Leer (HLLC) Riemann solver (88). Overall, the code achieves 3rd-order accuracy in space and 2nd-order accuracy in time. A detailed description and extensive tests of the hydrodynamic integration algorithm can be found in (68). Implementation of the reactive-diffusive extensions in ATHENA-RFX, along with the results of convergence studies, is discussed in detail in (69).

The reaction kinetics model represents thermonuclear burning in relativistic, degenerate plasmas present in stellar interiors during SNIa explosions (89). ATHENA-RFX implements a reaction network (often referred to as $\alpha$-chain) (50), which includes the triple- $\alpha, \alpha$-capture, and heavy-ion reactions for 13 isotopes: ${ }^{4} \mathrm{He}\left(\alpha\right.$-particle), ${ }^{12} \mathrm{C},{ }^{16} \mathrm{O},{ }^{20} \mathrm{Ne},{ }^{24} \mathrm{Mg},{ }^{28} \mathrm{Si},{ }^{32} \mathrm{~S}$, ${ }^{36} \mathrm{Ar},{ }^{40} \mathrm{Ca},{ }^{44} \mathrm{Ti},{ }^{48} \mathrm{Cr},{ }^{52} \mathrm{Fe}$, and ${ }^{56} \mathrm{Ni}$. Nuclear reaction rates are based on a standard tabulation (90) with screening corrections. The equation of state includes contributions from ideal ions, degenerate electrons, radiation, and electron-positron pairs (91). Thermal conduction includes both electron and photon components with degeneracy effects (92). To increase computational efficiency, both the equation of state and thermal conduction are tabulated and use bi-quadratic run-time interpolation. In thermonuclear flames, Lewis number $L e$, defined as the ratio of thermal conduction to species diffusion, $\rightarrow \infty$ and $\operatorname{Pr} \rightarrow 0$ (61). Thus, diffusion and viscosity were not included in the physical model, which makes this calculation effectively an Implicit Large-Eddy Simulation (70-73).

Treatment of general equations of state in ATHENA-RFX is implemented using the energy relaxation method (93). The stiff system of ordinary differential equations (ODE) for thermonuclear kinetics is integrated using a non-iterative, single-step, semi-implicit ODE integrator YASS (94). YASS does not employ any approximations to the Jacobian matrix, conserves species mass fractions and total energy explicitly, and balances numerical accuracy and computational efficiency.

The convergence studies performed for chemical flames show that the accuracy of the planar laminar flame solution is $1-2 \%$ for the resolution of 4 cells per laminar flame thickness $\delta_{L}$. Higher resolutions have little effect on the internal structure of flamelets, but can affect the flame wrinkling, and therefore affect the turbulent flame structure and dynamics. The turbulent flame speed decreases by $26 \%$ when the computational cell size $\Delta x$ decreases from $\delta_{L} / 8$ to $\delta_{L} / 16$, and by another $9 \%$ when $\Delta x$ decreases further to $\delta_{L} / 32$ (69). In this work, we use $\Delta x=\delta_{L} / 8$ for chemical flames.

For thermonuclear flames, we adopt $\Delta x=\delta_{L} / 4.68$ due to the higher computational cost of the equation of state, degenerate plasma transport, and thermonuclear reaction kinetics. This resolution allows us to reproduce the laminar flame speed with accuracy $\sim 10 \%$ and is similar to the resolutions used in prior fully-resolved simulations of thermonuclear flames $(72,73)$.

We also estimated the relative error, $S_{\text {err }}$ of integration that can accumulate in 3D numerical 
simulations $(95,96)$

$$
S_{\text {err }}=\sum_{i=1}^{3}\left(1 / N_{i}\right)^{k+1} \sqrt{n},
$$

where $N_{i}$ is the number of cells in each direction, $n$ is the number of time steps, and $k$ is the order of accuracy of a numerical scheme. For the simulation parameters listed in Table S1:

- $S_{\text {err }}=1.2 \times 10^{-7}$ for chemical flames $\quad(N=(8192,256,256), n=70000, k=3)$.

- $S_{\text {err }}=6.5 \times 10^{-9}$ for thermonuclear flames $(N=(8192,512,512), n=50000, k=3)$.

In both cases, the estimated integration error is extremely small and does not affect the simulation results. The accuracy of the numerical solution for our simulations is controlled by the finite numerical resolution of key physical features in the flow, such as flamelets and the turbulent cascade. We therefore rely on resolution tests for accuracy estimates.

\section{Calculations of the constant volume explosion properties}

To compute properties of a shock produced by a constant-volume explosion, we considered the initial composition (pure ${ }^{12} \mathrm{C}$ at $\rho=4 \times 10^{8} \mathrm{~g} \mathrm{~cm}^{-3}$ or $50 / 50{ }^{12} \mathrm{C} /{ }^{16} \mathrm{O}$ at $\rho=3 \times 10^{7} \mathrm{~g} \mathrm{~cm}^{-3}$ ) at the initial temperature $10^{9} \mathrm{~K}$. We solved a zero-dimensional problem of thermonumclear burning at $\rho=$ const and adiabatic conditions by integrating the $\alpha$-network to the equilibrium composition using YASS solver. The resulting pressure was used to compute shock properties by solving the Riemann problem. 
Table S1: Simulation parameters for chemical and thermonuclear flames

\begin{tabular}{|c|c|c|c|c|}
\hline & & Unit & Chemical & Thermonuclear \\
\hline Laminar flame width & $\delta_{L}$ & $\mathrm{~cm}$ & $4.154 \times 10^{-2}$ & $1.83 \times 10^{-4}$ \\
\hline Laminar flame speed & $S_{L}$ & $\mathrm{~cm} \mathrm{~s}^{-1}$ & 38.024 & $1.348 \times 10^{7}$ \\
\hline Fuel density & $\rho_{f}$ & $\mathrm{~g} \mathrm{~cm}^{-3}$ & $1.104 \times 10^{-3}$ & $4.0 \times 10^{8}$ \\
\hline Fuel temprature & $T_{f}$ & $\mathrm{~K}$ & 298.0 & $10^{8}$ \\
\hline Fuel pressure & $P_{f}$ & $\operatorname{ergs~} \mathrm{cm}^{-3}$ & $1.013 \times 10^{6}$ & $1.419 \times 10^{26}$ \\
\hline Sound speed in fuel & $c_{s, f}$ & $\mathrm{~cm} \mathrm{~s}^{-1}$ & $3.314 \times 10^{4}$ & $6.921 \times 10^{8}$ \\
\hline Domain width & $L$ & $\mathrm{~cm}$ & 1.328 & 0.02 \\
\hline Mesh size $(x \times y \times z)$ & & cells & $8192 \times 256 \times 256$ & $8192 \times 512 \times 512$ \\
\hline Flame widths per $L$ & $L / \delta_{L}$ & & 31.97 & 109.29 \\
\hline Cell size & $d x$ & $\mathrm{~cm}$ & $5.188 \times 10^{-3}$ & $3.906 \times 10^{-5}$ \\
\hline Cells per flame width & $\delta_{L} / d x$ & & 8.007 & 4.685 \\
\hline Turbulent velocity at scale $\delta_{L}$ & $U_{\delta}$ & $\mathrm{cm} \mathrm{s}^{-1}$ & $1.141 \times 10^{3}$ & $2.696 \times 10^{7}$ \\
\hline Normalized turb. velocity & $U_{\delta} / S_{L}$ & & 30.0 & 2.0 \\
\hline Turbulent velocity at scale $L$ & $U_{L}$ & $\mathrm{~cm} \mathrm{~s}^{-1}$ & $3.62 \times 10^{3}$ & $1.289 \times 10^{8}$ \\
\hline Integral velocity & $U_{l}$ & $\mathrm{~cm} \mathrm{~s}^{-1}$ & $2.402 \times 10^{3}$ & $8.592 \times 10^{7}$ \\
\hline Integral scale & $l$ & $\mathrm{~cm}$ & 0.388 & 0.006 \\
\hline Gibson scale & $L_{G}$ & $\mathrm{~cm}$ & $1.539 \times 10^{-6}$ & $2.288 \times 10^{-5}$ \\
\hline Inverse norm. Gibson scale & $\delta_{L} / L_{G}$ & & $2.7 \times 10^{4}$ & 8.0 \\
\hline Karlovitz number & $K a$ & & 164.32 & 2.883 \\
\hline Damkohler number & $D a$ & & 0.063 & 4.246 \\
\hline $\begin{array}{l}\text { Turbulent Mach number } \\
\text { in fuel at scale } L\end{array}$ & $M_{L}=U_{L} / c_{s, f}$ & & 0.109 & 0.186 \\
\hline Number of timesteps & $n$ & & 70,000 & 50,000 \\
\hline
\end{tabular}


Movie S1. Evolution of a turbulent flame and transition to a detonation in a stoichiometric $\mathrm{CH}_{4}$-air mixture for the same simulation as shown in Fig. 1. The movie shows the ray-tracing-based rendering of the isosurface of the fuel mass fraction $Y=0.5$. Colors correspond to the light intensity, which increases from red to yellow to white. The playback speed slows during the later stages of the flame evolution close to the moment of DDT.

Movie S2. Turbulence-driven spontaneous shock formation in a turbulent thermonuclear flame, for the same simulation as shown in Fig. 5. Shown is the isosurface of the ${ }^{12} \mathrm{C}$ mass fraction $Y=0.5$ along with the flame-generated pressure field. Isosurface is shown using ray-tracing-based rendering, with colors corresponding to the light intensity, which increases from red to yellow to white. Pressure is shown using ray-tracing-based volume rendering and a separate colormap given in the lower right corner. Pressure scale is in $\mathrm{ergs}^{-3}$. 


\section{References}

86. We note that, in principle, it could be possible to demonstrate this DDT process at lower densities and turbulent intensities using alternative modeling approaches, which do not aim to fully resolve the flame. In particular, by not resolving the flame, it could be possible to consider larger system sizes, which would be required for this DDT mechanism to operate at lower densities or in more ${ }^{12} \mathrm{C}$-poor mixtures. For instance, thickened flame techniques could represent one such possible strategy (87). It is important, however, for such modeling approaches to allow for the feedback process critical for this DDT mechanism to operate. In particular, they need to account for the changes in the local flame properties (speed and thickness) in response to the fuel compression during the runaway process, and the associated changes in the flame wrinkling by turbulence.

87. O. Colin, F. Ducros, D. Veynante, T. Poinsot. A thickened flame model for large eddy simulations of turbulent premixed combustion. Phys. Fluids. 12:1843-1863 (2000).

88. T. A. Gardiner, J. M. Stone. An unsplit Godunov method for ideal MHD via constrained transport in three dimensions. J. Comput. Phys. 227:4123-4141 (2008).

89. W. Hillebrandt, J. C. Niemeyer. Type Ia supernova explosion models. Annu. Rev. Astro. Astrophys. 38:191-230 (2000).

90. G. R. Caughlan, W. A. Fowler. Thermonuclear reaction rates V. At. Data Nucl. Data Tables 40:283 (1988).

91. F. X. Timmes, F. D. Swesty. The accuracy, consistency, and speed of an electron-positron equation of state based on table interpolation of the Helmholtz free energy. Astrophys. J. Supp. 126:501-516 (2000).

92. F. X. Timmes. Physical properties of laminar helium deflagrations. Astrophys. J. 528:913945 (2000).

93. F. Coquel, B. Perthame. Relaxation of energy and approximate Riemann solvers for general pressure laws in fluid dynamics. Soc. Industr. Appl. Math. J. Num. Anal. 35:2223-2249 (1998).

94. A. Khokhlov, I. Dominguez, C. Bacon, B. Clifford, E. Baron, P. Hoeflich, K. Krisciunas, N. Suntzeff, L. Wang. Three-dimensional simulations of thermonuclear detonation with $\alpha$-network: Numerical method and preliminary results. in Advances in computational astrophysics: Methods, tools, and outcome, R. Capuzzo-Dolcetta, M. Limongi, A. Tornambé, Eds. (Astron. Soc. Pacific Conf. Ser., 2012) vol. 453, pp. 107-114.

95. N.N.Smirnov, V.B.Betelin, R.M.Shagaliev, V.F.Nikitin, I.M.Belyakov, Yu.N.Deryuguin, S.V.Aksenov, D.A.Korchazhkin. Hydrogen fuel rocket engines simulation using LOGOS code. Int. J. Hydr. Ener. 39:10748-10756 (2014). 
96. N. N. Smirnov, V. B. Betelin,V. F. Nikitin, L. I. Stamov, D. I. Altoukhov. Accumulation of Errors in Numerical Simulations of Chemically Reacting Gas Dynamics. Acta Astronautica, 117:338-355 (2015) 